\title{
Fuzzy partitions: a way to integrate expert knowledge into distance calculations
}

\author{
Serge Guillaume ${ }^{\mathrm{a}}$, Brigitte Charnomordic ${ }^{\mathrm{b}}$, Patrice Loise $^{\mathrm{b}}$ \\ ${ }^{a}$ Irstea, UMR ITAP, BP 5095, 34196 Montpellier, France \\ ${ }^{b}$ INRA-SupAgro, UMR 729 MISTEA, F-34060 Montpellier, France
}

\begin{abstract}
This work proposes a new pseudo-metric based on fuzzy partitions (FPs). This pseudo-metric allows for the introduction of expert knowledge into distance computations performed on numerical data and can be used in various types of statistical clustering or other applications. The knowledge is formalized by a FP, in which each fuzzy set represents a linguistic concept. The pseudo-metric is designed to respect the FP semantics. The univariate case is first studied, and the pseudo-metric behavior is discussed using synthetic experiments. Then, a multivariate version is proposed as a Minkowski-like combination of univariate distances or semi-distances. The value of the proposal is illustrated with two real-world case studies in the fields of Biology and Precision Agriculture.
\end{abstract}

Keywords: semi-supervised, metric, expert knowledge, clustering, segmentation

\section{Introduction}

Clustering procedures are common statistical data analysis techniques used in many fields. Clustering can be of various types, mainly hierarchical algorithms and partitional algorithms. Different types of data are candidates for clustering, including numerical data, categorical data and more complex types of data, such as graphs and multimedia data. The clustering of numerical data is frequently addressed in the literature, with well-established methods such as $k$-means for partitioning. However, these methods do not

Email address: serge.guillaume@irstea.fr (Serge Guillaume)

Preprint submitted to International Journal of Information Sciences June 12, 2012 
allow for the incorporation of expert knowledge. Although challenging, the use of conjointly numerical data and expert knowledge may be a good way to guide clustering procedures using a semi-supervised approach and to improve clustering results.

Zadeh proposed the concept of the linguistic variable [30] to implement approximate concepts and reasoning. The goal was to ease the contribution of domain experts to system modeling. A fuzzy set, defined by its membership function, determines a symbol, called a linguistic concept, in the numerical space. The FP is then composed of the fuzzy sets corresponding to each of the linguistic labels. Other approaches, based on ontologies, can be used to formalize domain knowledge, as described, for instance, in [20]. However, these approaches essentially use symbolic information, and the numerical aspects are difficult to introduce.

The fuzzy set representation framework combines numerical and symbolic aspects and cannot be reduced to either of these paradigms.

Our objective in this paper is to take advantage of the linguistic variable concepts to use numerical and symbolic information related to the same variable together in the same clustering procedures, which is not typical in clustering. Clustering methods are designed to address several types of variables, e.g., numerical or symbolic variables, without combining different types of information for a given variable.

Most clustering or classification techniques are based on a function $d$, which determines how the dissimilarity of two elements is calculated, as follows: between individuals, between individual and group or between groups. Clustering results are sensitive to the choice of the dissimilarity function $[1,14]$.

The combination of numerical and symbolic information and its introduction into the dissimilarity function would allow for its use in any clustering method or, more generally, in any dissimilarity-dependent algorithm.

The dissimilarity function must have the properties of non-negativity and symmetry and satisfy the triangle inequality, and it can be proper (identity of indiscernibles) or semi-proper. In the first case, the dissimilarity function is a metric or distance. In the second case, the dissimilarity function is a pseudo-metric or semi-distance.

The most common metrics for numerical values are defined by the $L^{p}$ norms:

$$
\|x\|_{p}=\left(\left|x_{1}\right|^{p}+\ldots+\left|x_{n}\right|^{p}\right)^{\frac{1}{p}}
$$


where $x$ is a multidimensional vector $\left(x_{1}, x_{2} \ldots x_{n}\right)$. The classical case of the Euclidean norm is obtained for $p=2$.

Other distances can be used in multivariate problems in which variables are not independent, for instance, the Mahalanobis distance or the ChoquetMahalanobis operator [26].

Dissimilarity functions have also been defined for non-ordered categorical data, e.g., material $=\{$ plastic, metal, wood $\}$. The definition of a dissimilarity function is mainly based on the presence or absence of a given attribute. In [3], several similarity measures are compared. Some of these similarity measures consider the sample size, the number of values taken by the attribute and the frequencies of these values in the data set. The Lorentz curve allows for the ranking of multi-attribute categorical data [9]. To our knowledge, scant attention has been paid to similarity measures for ordered categorical data, such as price $=\{$ cheap, average, expensive $\}$. In this case, the distance between cheap and expensive must be higher than the distance between cheap and average and the distance between average and expensive. Ordered categorical data can be represented by linguistic variables, based on fuzzy sets, with each concept corresponding to a given fuzzy set.

Like many other concepts, the concept of distance has been generalized to fuzzy sets. In a survey [2], Bloch recalls the three types of fuzzy distances already defined: between two points in a fuzzy set, from a point to a fuzzy set and between two fuzzy sets. Many studies tackle this problem, including $[8,6,19,10,5]$. Certain proposals pay attention to the fulfillment of the triangle inequality [4]. Recent developments consider distance and similarity measures suited for new types of fuzzy sets, including interval-valued [31], intuitionistic [28] or hesitant [29] fuzzy sets, or try to define a new family of metrics for compact and convex fuzzy sets [27], which is useful for imprecise data analysis. Distances in rough set theory [18] and evidence theory [16] have also recently been analyzed in surveys.

Bloch [2] does not mention the distance between individuals within a FP. Such a distance has been little studied. Based on the membership degrees, such a distance would be valid for numerical measurements while also containing a symbolic component related to the granularity of information.

A FP carries semantics and knowledge about variable behavior. For the semantics attached to the partition-related concepts to be valid, the distance between two non-distinguishable elements must be equal to zero, which is the case for all of the elements within a given fuzzy set kernel. Furthermore, elements belonging to different concepts must always have a distance higher 
than elements belonging to the same concept.

In [11], a first attempt at designing this type of distance has been proposed to build a hierarchy of FPs. The initial partition was defined as a strong fuzzy partition (SFP) with as many fuzzy sets as distinct values for the given variable. Then, at each step, two adjacent fuzzy sets were merged into one new fuzzy set. The selected pair was the pair that yielded the minimum variation in the sum of distances for the entire training set. The objective of this process was to preserve the structure of the previous step.

The objective of the present work is different. The distance is not used to build a FP, but the FP is used to introduce expert knowledge into distance calculations.

Beginning with the preliminary study presented in [13], this paper proposes to take advantage of fuzzy set formalism to add a semi-supervised aspect to distance-based statistical procedures such as clustering. The semisupervision is performed by using available expert knowledge to superimpose linguistic concepts onto numerical data. This pretreatment is followed by the design of a pseudo-metric based on the FPs corresponding to the concepts and its use in clustering procedures. The procedure is thus an approach to combine numerical data and imperfect data resulting from human judgment.

The paper is organized as follows. Section 2 introduces a function based on FPs to calculate the dissimilarities between univariate points. Various areas are considered in the FP, and the function is designed accordingly to constitute a system of embedded functions. Then, the pseudo-metric properties are demonstrated. Section 3 discusses the particular case of SFPs. Section 4 studies the pseudo-metric behavior in depth and includes a comparison with the Euclidean distance, synthetic experiments and a real-world case study. A multivariate pseudo-metric is proposed in Section 5 that illustrates a real-world case study using partitioning methods. Section 6 provides conclusions.

\section{The proposed function and its properties}

The proposal applies to data in the unit interval $U=[0,1]$ and relies on FPs.

\subsection{Notations and constraints on the fuzzy partition}

Denote by $P$ the FP composed of $f$ fuzzy sets, defined by their respective membership functions (MFs) labeled $1,2 \ldots f$. 


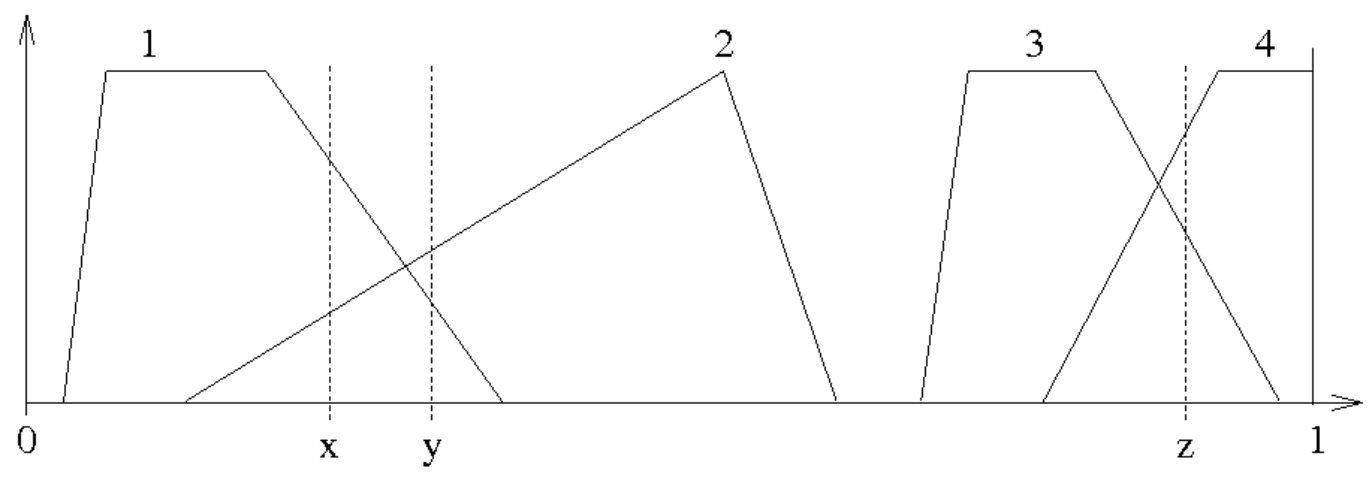

Figure 1: Example of FP and data points $\mathrm{x}, \mathrm{y}, \mathrm{z}$

Denote by $d_{*}(x, y)$ the proposed function for FP $P$.

Denote by $\mu_{i}(x)$ the membership degree of $x$ in the MF $i$.

Denote by $S_{i}=\left[S_{i}, \overline{S_{i}}\right]$ the support of MF $i$, defined by $\left\{x \mid \mu_{i}(x)>0\right\}$.

Denote by $K_{i}=\left[K_{i}, \overline{K_{i}}\right]$ the kernel of MF $i$, defined by $\left\{x \mid \mu_{i}(x)=1\right\}$.

By convention, $\underline{K_{0}}=\overline{K_{0}}=\overline{S_{0}}=0, \underline{K_{f+1}}=\overline{K_{f+1}}=\underline{S_{f+1}}=1$ and $\forall x \in U, \mu_{0}(x)=\mu_{f+1}(x)=0$.

The FP must respect the following constraints:

- it is composed of linear triangle or trapezoid MFs,

- any point belongs to two MFs at most, and

- $\forall i \in[1, f], \exists x$ such as $\mu_{i}(x)>0$ and $\mu_{j}(x)=0, \forall j \neq i$.

Rationale: The whole set of constraints is related to the MF distinguishability considerations, which is necessary for semantic reasons because the MFs represent linguistic concepts.

The linear triangle or trapezoid MFs are chosen for the sake of simplicity and because they have a bounded support. The limitation for any point to have at most non-null memberships in 2 MFs corresponds to the semantics of the FP. Each MF represents a concept, which is part of an ordered sequence. Therefore, any point should be either a prototype of a concept or a transition between two concepts. The last constraint requires that the kernel have at least one exclusive prototype, which prevents the support of a given MF from going beyond the kernel of the following MFs. 
An example of a partition satisfying the constraints is shown in Figure 1, and the notations are illustrated in Figure 2.

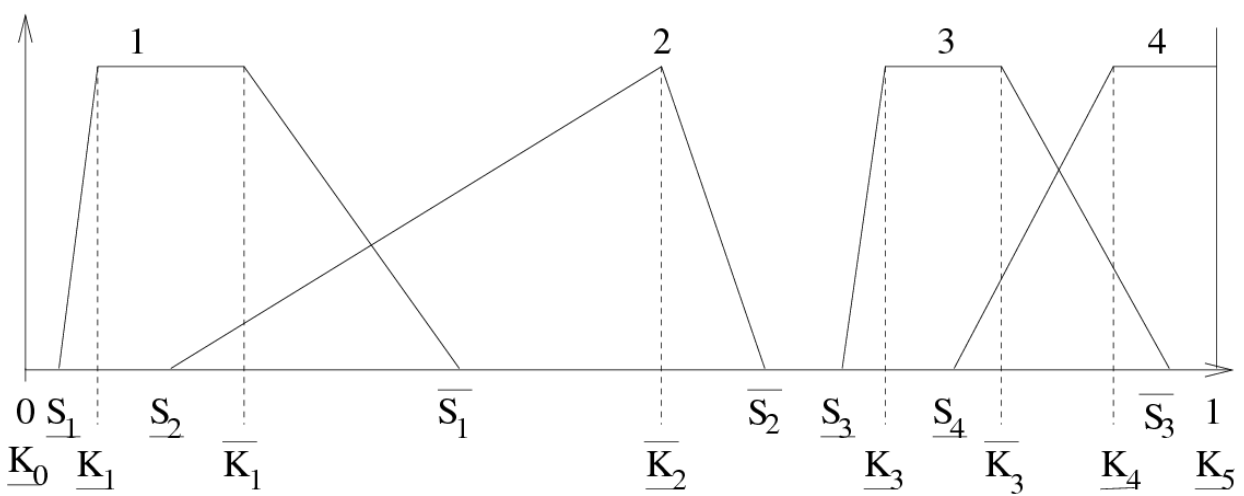

Figure 2: Notations for a FP

\subsection{Desirable behavior}

The proposed FP-based function combines numerical and symbolic elements. The numerical elements allow for the function to handle multiple memberships in transition zones, whereas the symbolic elements consider the granularity of the concepts associated with the fuzzy sets.

To ensure a smooth transition between concepts and a smooth behavior within a given $\mathrm{MF}$, the proposed function $d_{*}(x, y)$ must be continuous and non-decreasing when $x$ and $y$ move away from each other. The MFs must provide a structure to the distance calculation so that all points within a kernel have a null distance and so that $d_{*}(x, y)=|j-i|$ for $x \in K_{i}, y \in K_{j}$.

Semi-distance properties, which will be detailed in Section 2.5, are required for the proposed function. Thus, the function can be introduced into a wide range of applications including clustering and segmentation.

Many possible combinations of numerical and symbolic elements may be studied, but it is difficult to design a function that behaves like a semidistance. To explain our choice, we first study the case of data pairs lying within overlapping MF areas and then provide the general formula for the proposed function. 


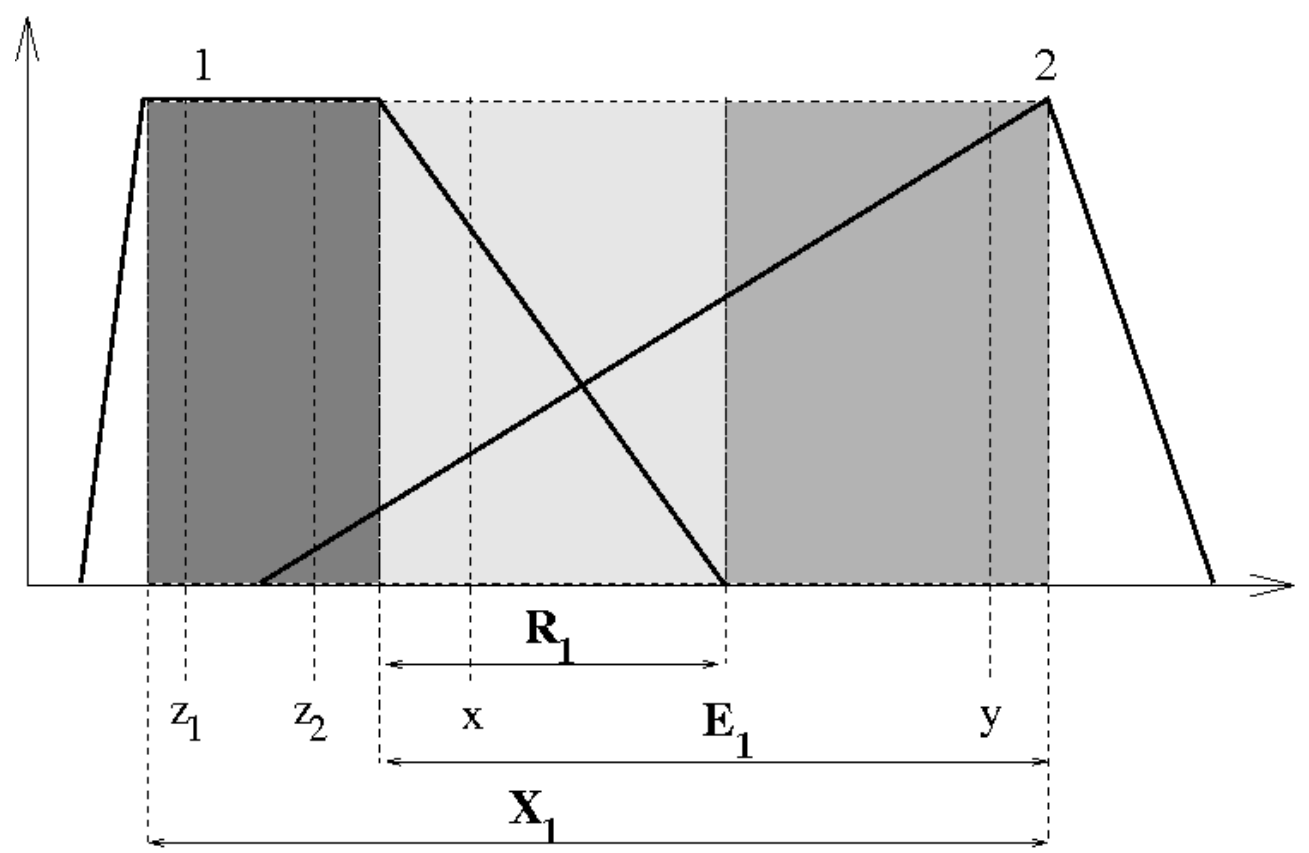

Figure 3: Various types of overlapping areas Restricted $\left(\mathbf{R}_{\mathbf{1}}\right)$, Embedded $\left(\mathbf{E}_{\mathbf{1}}\right)$, eXtended $\left(\mathbf{X}_{1}\right)$ 


\subsection{Decomposition into areas}

We define three types of overlapping areas, examples of which are shown in Figure 3:

Definition 2.1. (i) Let $X_{i}=\left[\underline{K_{i}}, K_{i+1}[\right.$, for $0 \leq i \leq f$, the eXtended overlapping area.

(ii) Let $E_{i} \subset X_{i}$, the Embedded overlapping area: $E_{i}=\left[\overline{K_{i}}, K_{i+1}[\right.$.

(iii) Let $R_{i} \subset E_{i}$, the Restricted overlapping area, defined by:

$$
R_{i}=\operatorname{cl}\left\{x \in E_{i} \mid \mu_{i}(x)>0, \mu_{i+1}(x)>0\right\}
$$

where $c l$ is the closure operator.

To summarize, we have $R_{i} \subset E_{i} \subset X_{i}$ and $X_{i}=E_{i} \cup K_{i}, \forall i \in[1, f]$.

In the example given in Figure 3, we have $R_{1}=\left[\overline{K_{1}}, \overline{S_{1}}\right]$, but this is not always true, depending on the support and kernel locations.

For each overlapping area and the total area, we propose distinct functions that, as we will demonstrate in Section 2.6, constitute a system of embedded pseudo-metrics.

\subsubsection{Within restricted overlapping areas}

To obtain a smooth behavior, the function is reduced to a numerical component $d_{n}$ that is based on the four membership degrees in the two MFs present within the restricted area:

Definition 2.2. Let $x, y \in[0,1]$ be any two points within the restricted overlapping area $R_{i}$. The numerical component $d_{n}$ is given by:

$$
\begin{aligned}
& \forall x \leq y \in R_{i}, d_{n}(x, y)=\mu_{i}(x) \mu_{i+1}(y)-\mu_{i+1}(x) \mu_{i}(y) \\
& \forall x>y \in R_{i}, d_{n}(x, y)=d_{n}(y, x)
\end{aligned}
$$

From Definition 2.2, we deduce the following property:

Proposition 2.1. The $d_{n}$ value satisfies $d_{n}(x, y)>0$ if $x \neq y$.

Proof: easily deduced from $\mu_{i}(x)>\mu_{i}(y), \mu_{i+1}(y)>\mu_{i+1}(x)$. 


\subsubsection{Within embedded overlapping areas}

The embedded overlapping area is composed of a core, defined previously as the restricted overlapping area, surrounded (or not) by two subareas in which only one MF is present. Because the number of non-null membership degrees to consider varies depending on the location of the fuzzy set bounds, it is easier to define the $d_{0}$ function, which is valid within the embedded overlapping areas, as a sum of three terms, each of which corresponds to one of these subareas.

Definition 2.3. Let $x, y \in[0,1]$ be any two points within the embedded overlapping area $E_{i}$. The numerical component $d_{0}$ is given for all $x \leq y \in E_{i}$,:

$$
\begin{aligned}
& \qquad \begin{aligned}
d_{0}(x, y) & =\frac{1}{N_{i}}\left[\mu_{i}(x)-\mu_{i}\left(\min \left(y, \max \left(x, \underline{S_{i+1}}\right)\right)\right)\right. \\
& +d_{n}\left(\max \left(x, \underline{S_{i+1}}\right), \min \left(y, \overline{S_{i}}\right)\right) \\
& \left.+\mu_{i+1}(y)-\overline{\mu_{i+1}}\left(\max \left(x, \min \left(y, \overline{S_{i}}\right)\right)\right)\right] \\
\text { with } N_{i}=1- & \mu_{i}\left(\underline{S_{i+1}}\right)+d_{n}\left(\underline{S_{i+1}}, \overline{S_{i}}\right)+1-\mu_{i+1}\left(\overline{S_{i}}\right), 1 \leq i \leq f-1 \\
N_{0}=N_{f} & =1
\end{aligned}
\end{aligned}
$$

and for all $x>y \in E_{i}, d_{0}(x, y)=d_{0}(y, x)$.

Remarks:

1. Definition 2.2 is extended to data points $x, y \notin R_{i}$, so that, in this case, $d_{n}(x, y)=0$.

2. In Equation (2), min and max are introduced to comply with the various possible locations of the data points within the $R_{i}$ and $E_{i}-R_{i}$ subareas.

3. The normalization coefficient $N_{i}$ guarantees that $\forall x, y, d_{0}(x, y) \in[0,1]$.

Example: Let us consider the values $x, y$ shown in Figure 3. In this case, the first element of $d_{o}(x, y)$ is equal to zero, the second element is equal to $d_{n}\left(x, \overline{S_{1}}\right)$ and the last element is equal to $\mu_{2}(y)-\mu_{2}\left(\overline{S_{1}}\right)$.

\subsubsection{Within extended overlapping areas}

By convention, all points that belong to a given MF kernel, such as $z_{1}$ and $z_{2}$ in Figure 3, are considered to have a null distance. This convention is necessary to respect the fuzzy set semantics. Thus, 


$$
\forall i \in[1, f], \forall x, y \in\left[\underline{K_{i}}, \overline{K_{i}}\right] \Rightarrow d_{*}(x, y)=0
$$

Therefore, $d_{*}\left(K_{i}, \overline{K_{i}}\right)=0$. Hence, in extended overlapping areas, we define the $d_{1}$ function using the $d_{0}$ function in embedded overlapping areas:

Definition 2.4. Let $x, y \in[0,1]$ any two points within the extended overlapping area $X_{i}$. The numerical component $d_{1}$ is given by:

$$
\forall x, y \in X_{i}, d_{1}(x, y)=d_{0}\left(\max \left(x, \overline{K_{i}}\right), \max \left(y, \overline{K_{i}}\right)\right)
$$

\subsection{Proposed function in the whole domain}

When the data points do not lie within the same overlapping area, as is the case for points $x$ and $z$ in Figure 4, the formula is more complex and contains a numerical component $d_{1}$, which reflects the relative location within the extended area to which each point belongs, and a symbolic component, which accounts for the number of MFs between the points.

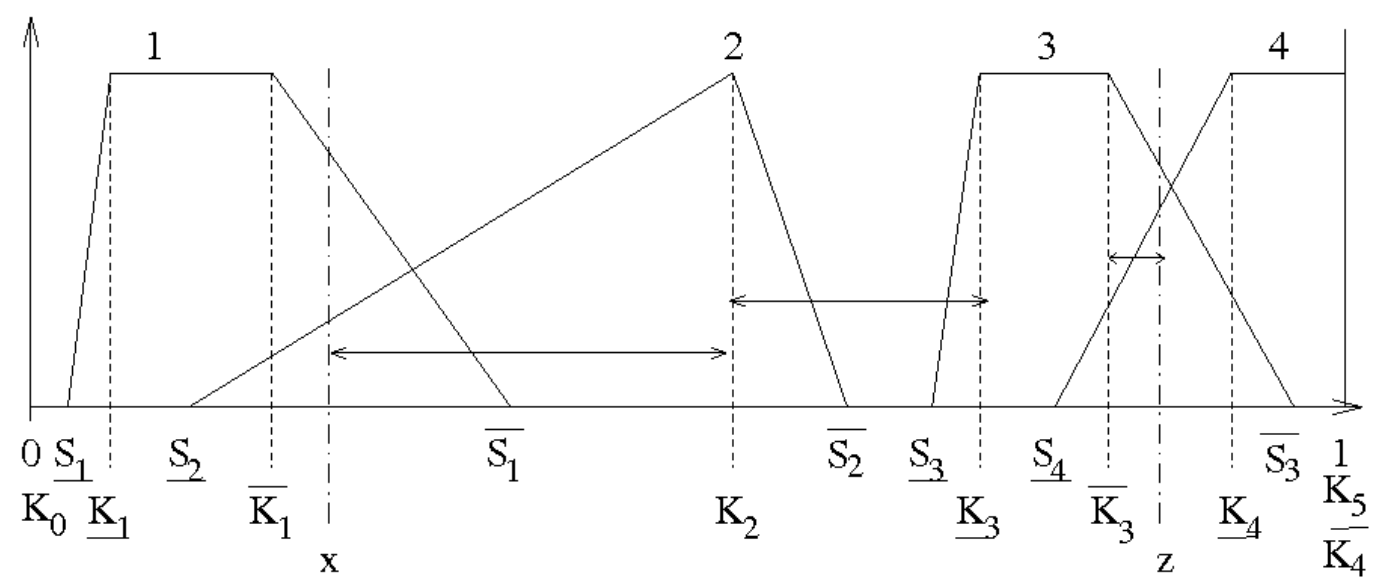

Figure 4: FP-based univariate function calculation; arrows represent the partial distances to be combined

We denote by $I(x)$ the function such that:

$$
\forall i \in[0, f], x \in X_{i} \Leftrightarrow I(x)=i
$$

The function $d_{2}(x, z)$ is calculated as a sum of partial elements associated with various areas: the area from $x$ to the kernel of the $(I(x)+1)$ th $\mathrm{MF}$, the 
area between the kernels of the $(I(x)+1)$ th and the $I(z)$ th $\mathrm{MF}$ and the area from the kernel of the $I(z)$ th MF to $z$.

For semantic considerations, the distance between the two kernels of MFs $I(x)$ and $I(z)$ is assumed to be the distance between the two corresponding linguistic concepts. It is a symbolic element, equal to $d_{s}(I(x), I(z))=\mid I(z)-$ $I(x) \mid$, which does not depend on the MF bounds. The following formula is proposed:

Definition 2.5. Let $x, z \in[0,1]$ be any two points. For all $x \leq z$,

$d_{*}(x, z)=\left\{\begin{array}{lr}d_{1}(x, z) & \text { if } I(x)=I(z) \\ d_{1}\left(x, \underline{K_{I(x)+1}}\right)+d_{s}(I(x)+1, I(z))+d_{1}\left(\underline{K_{I(z)}}, z\right) & \text { if } I(x)<I(z)\end{array}\right.$

where $d_{s}(i, j)=|j-i|, \forall i, j \in[1, f-1]$.

For all $x>z, d_{*}(x, z)=d_{*}(z, x)$.

If $\mathrm{f}$ is large, the term using $d_{s}$ becomes predominant, while the terms involving $d_{1}$ have a relatively limited influence, with $d_{1}$ being between 0 and 1. This is expected to reflect the expert knowledge.

The various parts of the sum in Equation (4) are illustrated by the arrows in Figure 4. In this case, we have $I(x)=1$ and $I(z)=3$.

According to Equation (4), the symbolic component is zero when $I(z)=$ $I(x)+1$. In this case, $x$ and $z$ are located on both sides of a given fuzzy set kernel.

The $d_{*}$ distance could be used as such. The upper bound of the distance may take the following values, $f-1, f, f+1$, according to the partition edges. The dependence on $f$ can be a problem if one wants to compare FPs of various sizes. To address this issue and achieve a better commensurability between the different terms, normalization is proposed. The general formula becomes

$$
d_{P}(x, y)=\frac{d_{*}(x, y)}{f-1+d_{0}\left(0, \underline{K_{1}}\right)+d_{0}\left(\overline{K_{f}}, 1\right)}
$$

where $f$ is the number of fuzzy sets and $P$ stands for a given FP. In the equation, $d_{0}\left(0, \underline{K_{1}}\right)$ and $d_{0}\left(\overline{K_{f}}, 1\right)$ may take 0 or 1 values.

This normalization may hide the level of granularity chosen by the expert. If no comparison is required between FPs of various sizes, $d_{*}$ should be preferred. 
The properties of dissimilarities, semi-distances and distances are now recalled before demonstrating which of the properties are satisfied by the proposed function $d_{P}$.

\subsection{Distance properties}

A function $d$ is a dissimilarity if

$$
\forall x, y \in U, \quad\left\{\begin{array}{l}
d(x, y) \geq 0 \\
d(x, x)=0 \\
d(x, y)=d(y, x)
\end{array}\right.
$$

A dissimilarity is semi-proper if $d(x, y)=0 \Rightarrow \forall w, d(x, w)=d(y, w)$, and it is proper if $d(x, y)=0 \Rightarrow x=y$.

A semi-distance is a dissimilarity that verifies the triangle inequality:

$$
\forall x, y, z \in U, \quad d(x, y) \leq d(x, z)+d(z, y)
$$

A proper semi-distance is called a distance. In this paper, the goal is not to ensure the proper character because all kernel elements must be indistinguishable. The expected properties are those of a semi-distance.

\subsection{Checking properties}

Let us examine the cases that correspond to the different areas, where Equations (1), (2), (3), (4) or (5) are applicable.

1. Properties of the function $d_{n}$ on restricted overlapping areas Let us prove that $d_{n}$ is a distance.

Proposition 2.2. The function $d_{n}$ is a distance. It is symmetric by construction.

Proof: $d_{n}$ is obviously a dissimilarity. We have to prove that $d_{n}$ is proper and satisfies the triangle inequality.

(i) $d_{n}$ is proper: if we assume $d_{n}(x, y)=0$, then $\mu_{i}(x) \mu_{i+1}(y)=\mu_{i}(y) \mu_{i+1}(x)$.

As $\overline{\mu_{i}(x) \geq \mu_{i}(y)}$ and $\mu_{i+1}(y) \geq \mu_{i+1}(x)$, we deduce that $\mu_{i}(x)=\mu_{i}(y)$ and $\mu_{i+1}(y)=\mu_{i+1}(x)$. Therefore, $x=y$.

(ii) $d_{n}$ satisfies the triangle inequality:

Let $a=S_{i+1}$ and $b=\overline{S_{i}}$. Because the MFs are linear functions, $\mu_{i}(x)=$ $s_{i}(b-x)$, where $s_{i}$ is a positive coefficient. Similarly, $\mu_{i+1}(x)=s_{i+1}(x-$ $a)$, where $s_{i+1}>0$. We consider three cases: 
(iia) $z<x<y$. It is sufficient to show that $d_{n}(x, y) \leq d_{n}(z, y)$ :

$$
\begin{aligned}
d_{n}(z, y)-d_{n}(x, y) & =\left(\mu_{i}(z)-\mu_{i}(x)\right) \mu_{i+1}(y)-\mu_{i}(y)\left(\mu_{i+1}(z)-\mu_{i+1}(x)\right) \\
& =s_{i} s_{i+1}[(x-z)(y-a)-(z-x)(b-y)] \\
& =s_{i} s_{i+1}(x-z)(b-a) \geq 0
\end{aligned}
$$

(iib) $x<z<y$. The inequality (6) to be verified becomes

$$
\begin{aligned}
\forall x, y, z \in R_{i}, \mu_{i}(x) \mu_{i+1}(y)-\mu_{i}(y) \mu_{i+1}(x) & \leq \mu_{i}(x) \mu_{i+1}(z)-\mu_{i}(z) \mu_{i+1}(x) \\
& +\mu_{i}(z) \mu_{i+1}(y)-\mu_{i}(y) \mu_{i+1}(z)
\end{aligned}
$$

which can be rewritten as

$$
\left(\mu_{i}(y)-\mu_{i}(z)\right)\left(\mu_{i+1}(y)-\mu_{i+1}(x)\right) \leq\left(\mu_{i}(x)-\mu_{i}(y)\right)\left(\mu_{i+1}(z)-\mu_{i+1}(y)\right)
$$

Therefore, using $\mu_{i}$, the previous inequality becomes

$$
(z-y)(y-x) \leq(y-x)(z-y) .
$$

Thus, the triangle inequality is proven.

(iic) $x<y<z$, which has the same proof as that for case a.

2. Properties of the $d_{0}$ function on embedded overlapping areas.

The definition of $d_{0}$ is based on the addition of the Euclidean distance and the distance $d_{n}$ on disjoint intervals. Therefore, $d_{0}$ is a distance.

3. Properties of the $d_{1}$ function on extended overlapping areas

In the case of kernels reduced to a single point, the definition of $d_{1}$ reduces to the distance $d_{0}$. Therefore, $d_{1}$ is a distance. Otherwise, $d_{1}$ is not proper but is only semi-proper. Therefore, in general, $d_{1}$ is a semi-distance.

4. Properties of the $d_{*}$ function (and then $d_{P}$ )

The definition of $d_{*}$ is based on the addition of the numerical semi -distance $d_{1}$ and the symbolic distance $d_{s}$ on disjoint intervals. Therefore, $d_{*}$ (and therefore $d_{p}$ ) is a semi-distance.

\section{The particular case of strong fuzzy partitions}

A SFP, described by $f$ membership functions on the universe $U$, fulfills the following condition:

$$
\forall x \in U, \quad \sum_{i=1}^{f} \mu_{i}(x)=1
$$




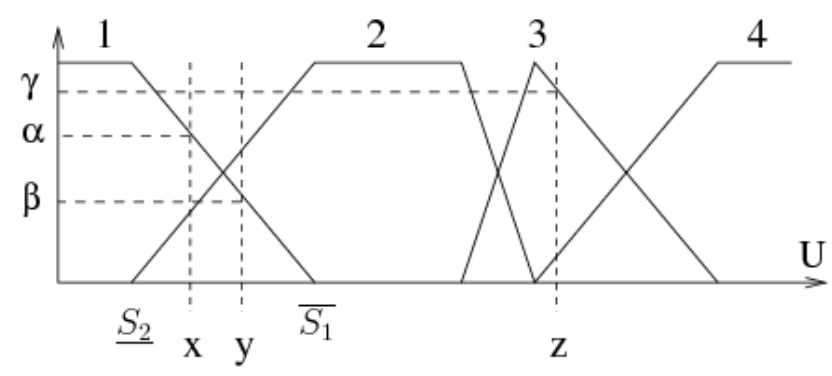

Figure 5: Example of a SFP

An example of SFP is shown in Figure 5.

Because of the SFP structure, the following relationship always holds:

$$
\forall i \in[1, f-1], \forall x \in U, \quad \mu_{i}(x)+\mu_{i+1}(x)=1
$$

Equation (1) becomes:

$$
d_{n}(x, y)=\mu_{i}(x)-\mu_{i}(y)
$$

Thus, for SFPs, the numerical component is defined only by the membership degrees to a single MF, the first MF in the partition order. For the example shown in Figure $5, d_{n}(x, y)=\alpha-\beta$.

Within the restricted overlapping area,

$$
\forall x \in R_{i}, \mu_{i}(x)=1-\frac{x-\underline{S_{i+1}}}{\overline{S_{i}}-\underline{S_{i+1}}}
$$

Thus, given two points in $R_{i}, d_{n}(x, y)=\frac{|y-x|}{\overline{S_{i}}-S_{i+1}}$ is directly proportional to the Euclidean distance between the two points.

Because of the SFP structure, $R_{i} \equiv E_{i}$, and the corresponding function satisfies $d_{0} \equiv d_{n}$.

After further simplification, Equation (4) becomes

$$
\forall x, y \in U, d_{*}(x, y)=\left|\mu_{I(x)}(x)-\mu_{I(y)}(y)+I(y)-I(x)\right|
$$


The P-based univariate pseudo metric is:

$$
\forall x, y \in U, d_{P}(x, y)=\frac{\left|\mu_{I(x)}(x)-\mu_{I(y)}(y)+I(y)-I(x)\right|}{f-1}
$$

Equivalent definition from a function

The SFP-based proposed pseudo-metric can also be designed using a function.

Let us introduce the function $\mathrm{P}$ :

$$
P(x)=I(x)-\mu_{I(x)}(x)
$$

$P$ is a positive non-decreasing function of $x$ and is increasing in overlapping zones.

$d_{P}(x, y)$ can then be written as

$$
d_{P}(x, y)=\frac{|P(y)-P(x)|}{f-1}
$$

This formulation, which shows that the use of SFPs is an elegant alternative to data transformation, also makes it easier to check the properties of SFPbased pseudo-metrics.

The quality of SFPs has been previously highlighted in the past. Pedrycz [22] noted that SFPs are error-free reconstructions for Sugeno-type systems using centroid defuzzification:

$$
\forall x \in U, \quad \psi[\phi(x)]=x
$$

where $\phi$ is the input space transform and $\psi$ is the output space transform.

Euclidean distance and regular fuzzy partitions

Definition 3.1. A regular fuzzy partition is composed of triangular MFs, with equidistributed kernel centers $K_{1} \ldots K_{f}$, with $K_{i}=\overline{S_{i-1}}=\underline{S_{i+1}}$.

A regular fuzzy partition with six MFs is shown in Figure 6. 


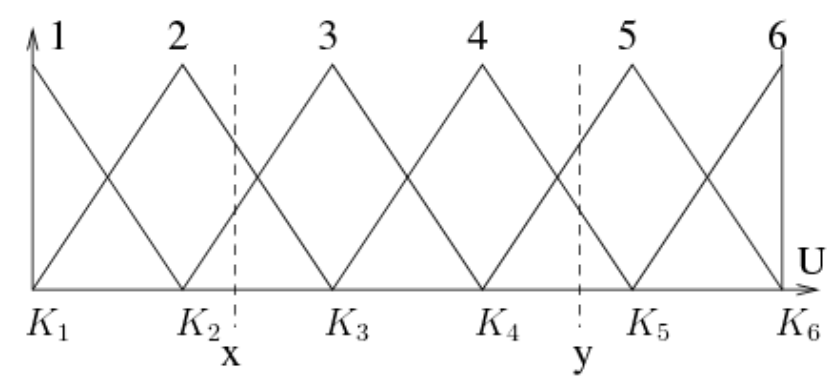

Figure 6: A regular fuzzy partition

For this particular case, we show that the proposed pseudo-metric is a metric and that it yields the same result as the Euclidean distance, regardless of the number of terms in the partition.

The regular distribution of the fuzzy set centers allows for the simplification of Equation (3) because data are normalized in the unit interval:

$$
K_{1}=\underline{S_{1}}=0 \text { and } \forall i>1, \quad K_{i}=\underline{S_{i+1}}=\overline{S_{i}}=\frac{i-1}{f-1}
$$

which yields $\overline{S_{i}}-\underline{S_{i+1}}=\frac{1}{f-1}$.

The function $\mathrm{P}$, defined in Equation (9), becomes $P(x)=(f-1) x$, and finally, $d(x, y)=|y-x|$.

The Euclidean distance and the regular SFP-based metric are identical when the data are in the unit interval.

This equivalence results because the distance proposed in Equation (5) distorts the Euclidean distance according to the two following points: the symbolic distance between concepts and the indistinguishability of the kernel elements. In the case of a regular SFP, these two characteristics disappear because all kernels are reduced to single points and are equidistant.

\section{Behavior and illustration in the univariate case}

This section investigates the univariate pseudo-metric behavior and illustrates its utility. This section compares the univariate pseudo-metric with the Euclidean distance and provides two examples of use of the univariate 
pseudo-metric: synthetic experiments using hierarchical clustering and a realworld case study with a segmentation algorithm.

\subsection{Comparison with the Euclidean distance}

Let us highlight two important properties of the proposed FP-based pseudo-metric.

First, the FP-based pseudo-metric value is always zero for any two points that belong to the same fuzzy set kernel.

Second, the $d_{P}$ pseudo-metric is typically different from the Euclidean distance, and the deviations depend on the fuzzy set slope and kernel width. These deviations may produce a different ranking compared with the Euclidean distance, as shown in Figure 7 . The three points $x, y, z$ have coordinates $0.2,0.3,0.5$. According to the Euclidean distance, denoted by $d_{E}$,

$$
d_{E}(x, y)=0.1<d_{E}(y, z)=0.2
$$

and when the FP-based pseudo-metric is used,

$$
d_{P}(y, z)=0.067<d_{P}(x, y)=0.133
$$

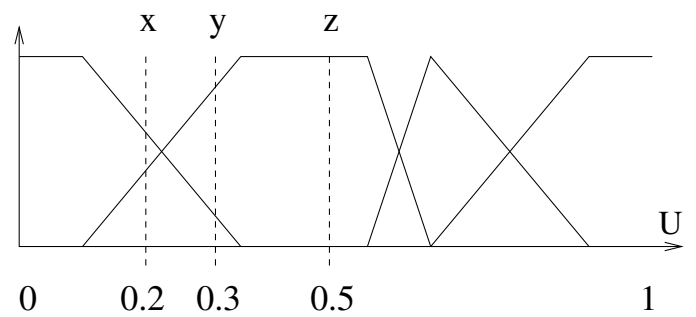

Figure 7: An example of an inversion compared to the Euclidean distance

\subsection{General behavior}

The proposed pseudo-metric behavior is likely to depend on two main characteristics of the FP: the number of linguistic concepts, which affects the symbolic component, and the size of the different kernels, with the wider the kernels having more numerous indistinguishable values.

It would be difficult to conduct a separate study of the influence of the various parameters because the parameters influence one another. Therefore, 


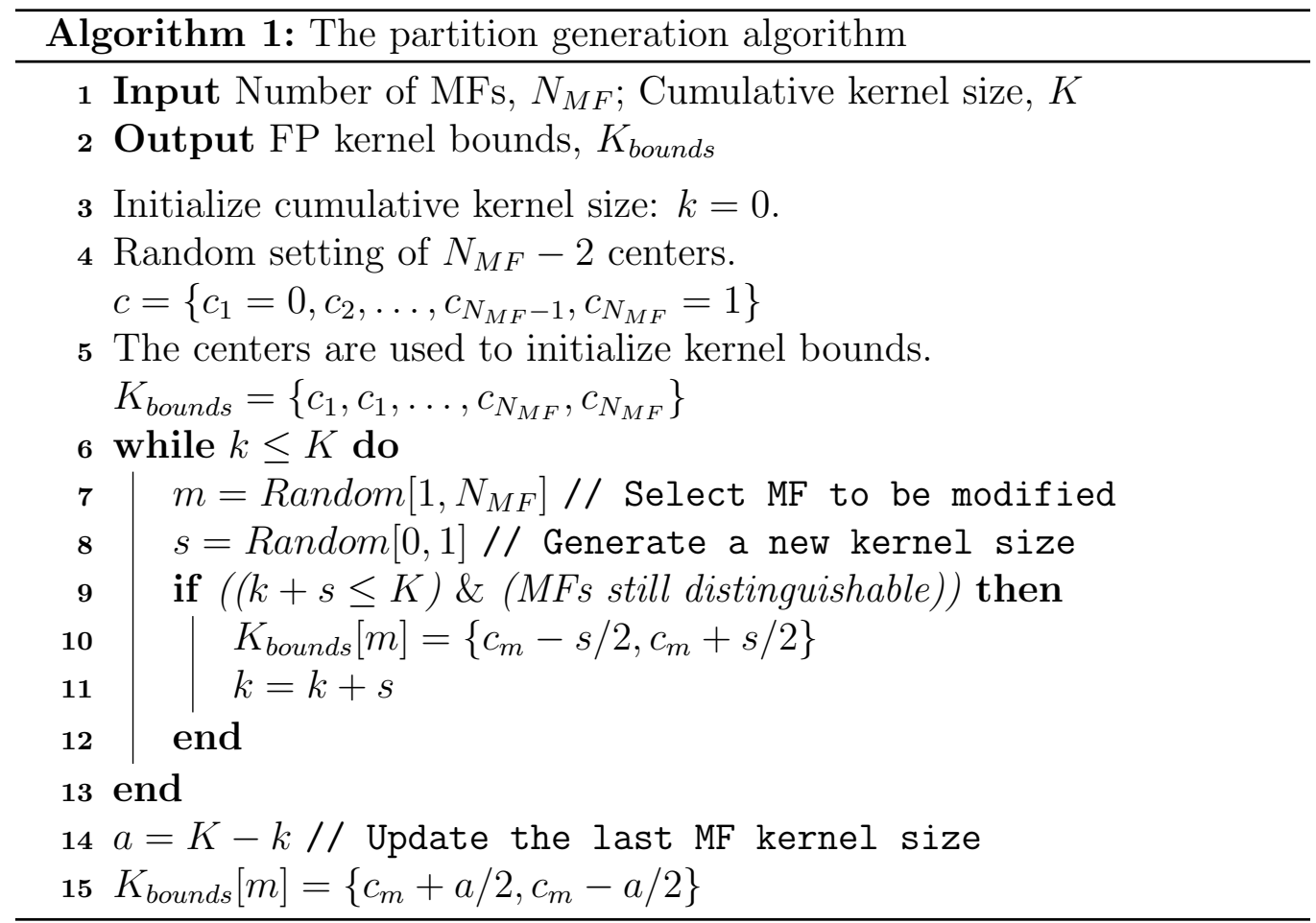


numerical simulations have been undertaken to investigate the general behavior of the distance. Various SFPs have been randomly generated according to Algorithm 1.

The two input parameters of the generation algorithm are $N_{M F}(>2)$, the number of MFs, and $K$, the cumulative kernel width over the partition. The first and last MF centers are set to 0 and 1 to address the case in which the cumulative size of kernels is zero, i.e., when all MFs are triangular in shape. The distinguishability constraints mentioned in Algorithm 1 are such that when an MF is modified, its new lower (upper) kernel bound is still higher (lower) than the upper (lower) kernel bound of the previous MF.

The partitions are characterized using a regularity index, defined as follows:

Definition 4.1. Let $P$ be any partition composed of $N_{M F}$ trapezoidal or triangular MFs over the same definition domain $D$. The ith MF kernel of $P$ is denoted by $\left[\underline{K_{i}}, \overline{K_{i}}\right]$.

The regularity index of $P$ is defined by comparison with a regular fuzzy partition RP of size $N_{M F}$ (see Definition 3.1). The ith MF kernel of RP is denoted by $c_{i}$.

$$
\begin{aligned}
& R I(P)=\frac{1}{\sqrt{N_{M F}-2}} \sum_{i=1}^{N_{M F}} d^{i}\left(c_{i}, P\right), \text { with } \\
& d^{i}(c, P)=\left\{\begin{array}{l}
0, \quad \text { if } c \in\left[\underline{K_{i}}, \overline{K_{i}}\right] \\
\min \left(d_{E}\left(c, \underline{K_{i}}\right), d_{E}\left(c, \overline{K_{i}}\right)\right), \text { otherwise }
\end{array}\right.
\end{aligned}
$$

The FP is designed to distort the Euclidean distance. To evaluate the impact of a given partition on this distortion, $N$ points are equally spaced in the unit interval, and the following distortion estimation is computed over all pairs of points.

$$
D D E(P)=\frac{1}{\sqrt{\frac{1}{2}\left(N^{2}-N\right)}} \sqrt{\sum_{j=1}^{N} \sum_{k=j+1}^{N}\left(d_{E}\left(x_{j}, x_{k}\right)-d_{P}\left(x_{j}, x_{k}\right)\right)^{2}}
$$

The random partition generation is performed according to Algorithm 1 for all combinations of $N_{M F}$ from 4 to 10 and $K \in\{0,0.05,0.1,0.15,0.2,0.25,0.3\}$; the number of points $\mathrm{N}$ is set to 100 , and the entire procedure is repeated 100 times. Numerical experiments showed that $R I(P)$ has a similar expectation, independent of the value of $N_{M F}$, calculated over the sample experiments 
Author-produced version of the article published in Information Sciences, 2013, 245, 76-95.

The original publication is available at http://www.sciencedirect.com

DOI : 10.1016/j.ins.2012.07.045

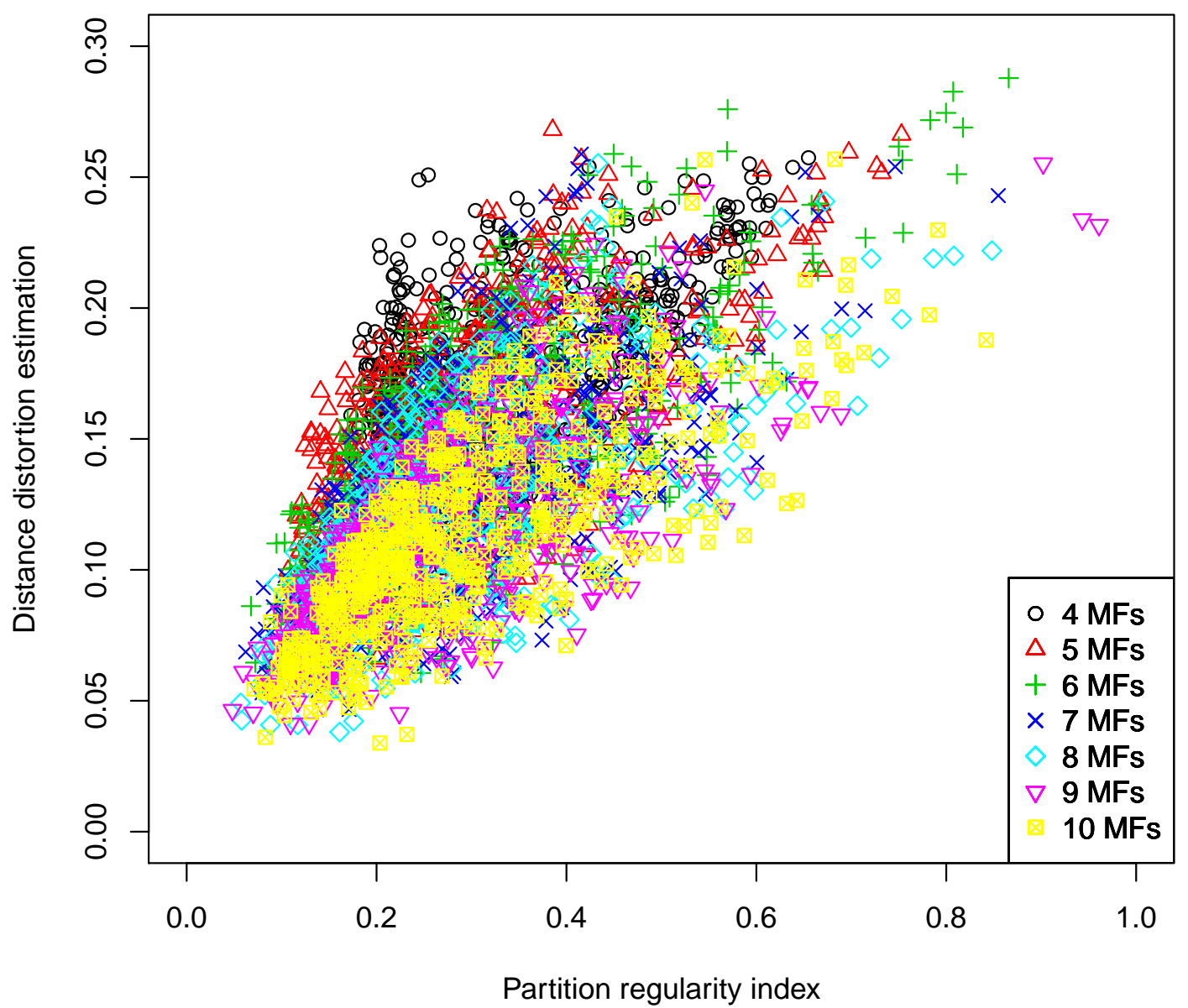

Figure 8: Illustration of the general behavior 
$\left(0.268<E[R I(P)]<0.297\right.$ for $N_{M F}$ between 5 to 10$)$. This justifies the chosen normalization in Equation (11).

Figure 8 shows the results of the 100 experiments for each of the configurations, with the regularity index $R I(P)$ as the abscissa and the distance distortion estimation $D D E(P)$ as the ordinate. The color and shape of the points correspond to a given number of MFs, regardless of the cumulative size of the kernels.

The linear regressions of $D D E$ versus $R I$, calculated independently for a given number of MFs, yield similar results: the slope, which varies between 0.202 and 0.238 for $N_{M F}$ between 5 and 10, and the $R^{2}$ coefficient are similar. The model that predicts the distance distortion according to the partition regularity index explains half of the variance $\left(R^{2} \approx 0.5\right.$, for $N_{M F}$ between 5 and 10), meaning that because of proper normalization, the distance behavior is not significantly influenced by the number of MFs in the partition. Instead, the distance behavior is influenced mostly by its regularity. The proposed index is one way to estimate this regularity and could certainly be improved. The case of $N_{M F}=4$ differs slightly, with a lower slope of 0.155 , a greater expectation (0.332) and a smaller $R^{2}=0.279$.

These numerical experiments illustrate a general trend because the partitions are randomly generated with little constraint and therefore vary significantly. The next section highlights the influence of the kernel size for partitions in which the deformation is more limited.

\subsection{Synthetic case study}

We will now examine the pseudo-metric behavior using a synthetic case study with univariate hierarchical clustering. A bottom up approach is used: each observation begins in its own cluster, and pairs of clusters are merged as we move up the hierarchy. At each step, the selected pair of clusters to be merged is the pair that yields the minimum increase of within-group variance. The dendrogram can be analyzed to determine the composition of the clusters at a given hierarchical level.

The considered synthetic variable is wind speed. The speed, measured in either $m p h$ or $\mathrm{km} / \mathrm{h}$, is usually expressed through a partition endowed with natural semantics: the Beaufort scale ${ }^{1}$. Thirteen degrees are defined in the range of $[0-120] \mathrm{km} / \mathrm{h}$. Higher degrees have wider ranges of speed.

\footnotetext{
${ }^{1}$ http://en.wikipedia.org/wiki/Beaufort_scale
} 
For example, the range of degree 2, labeled Light Breeze, is $[5.6-11] \mathrm{km} / \mathrm{h}$, whereas the range of degree 5, Fresh Breeze, is [29-38] $\mathrm{km} / \mathrm{h}$.

To simplify the study, the degrees are placed in four groups, each of which consists of approximately three degrees. The group characteristics are summarized in Table 1.

Table 1: The four groups of Beaufort degrees

\begin{tabular}{|l|cccc|}
\hline Groups & 1 & 2 & 3 & 4 \\
\hline Label & Calm & Breeze & Wind & Storm \\
Degrees & $0-2$ & $3-5$ & $6-9$ & $10-12$ \\
Interval $(\mathrm{km} / \mathrm{h})$ & $0-11$ & $12-38$ & $39-88$ & $89-\infty$ \\
Center $(\mathrm{km} / \mathrm{h})$ & 5 & 25 & 64 & 120 \\
\hline
\end{tabular}

The impact of the pseudo-metric behavior on the hierarchical clustering procedure, implemented as hclust in the $R$ environment[23], is analyzed. The results are highly sensitive to two factors: the choice of the metric between individuals and the method chosen for agglomerating the clusters. In the present study, the centroid agglomeration method is used as the method for agglomerating the clusters, and the metric used between individuals is a series of several metrics or pseudo-metrics that are Euclidean or FP-based. The hierarchical clustering results change accordingly and are studied at the hierarchical level corresponding to the four clusters and for various sample sizes: 100, 200, 500 and 1000.

For each sample size, ten samples have been randomly generated according to a Gamma law with parameters 3 (shape) and 12 (scale), and five pseudo-metrics are studied.

These FPs have been designed as SFPs and have been built with triangular or trapezoidal MF shapes. A triangular MF-based FP is designed, with kernel locations given in row 4 of Table 1, and trapezoidal MFs are added at the partition edges to form an SFP. Various trapezoidal MF-based FPs are studied, in which each MF kernel is built with a width corresponding to a given fraction: 25, 50 and $95 \%$ of the Wind speed interval reported in the third row of Table 1. All of the FPs are SFPs.

Let us first examine the results obtained with a sample size equal to 500 . Comments about the behavior with other sample sizes are presented at the end of the section. 
Author-produced version of the article published in Information Sciences, 2013, 245, 76-95.

The original publication is available at http://www.sciencedirect.com

DOI : 10.1016/j.ins.2012.07.045

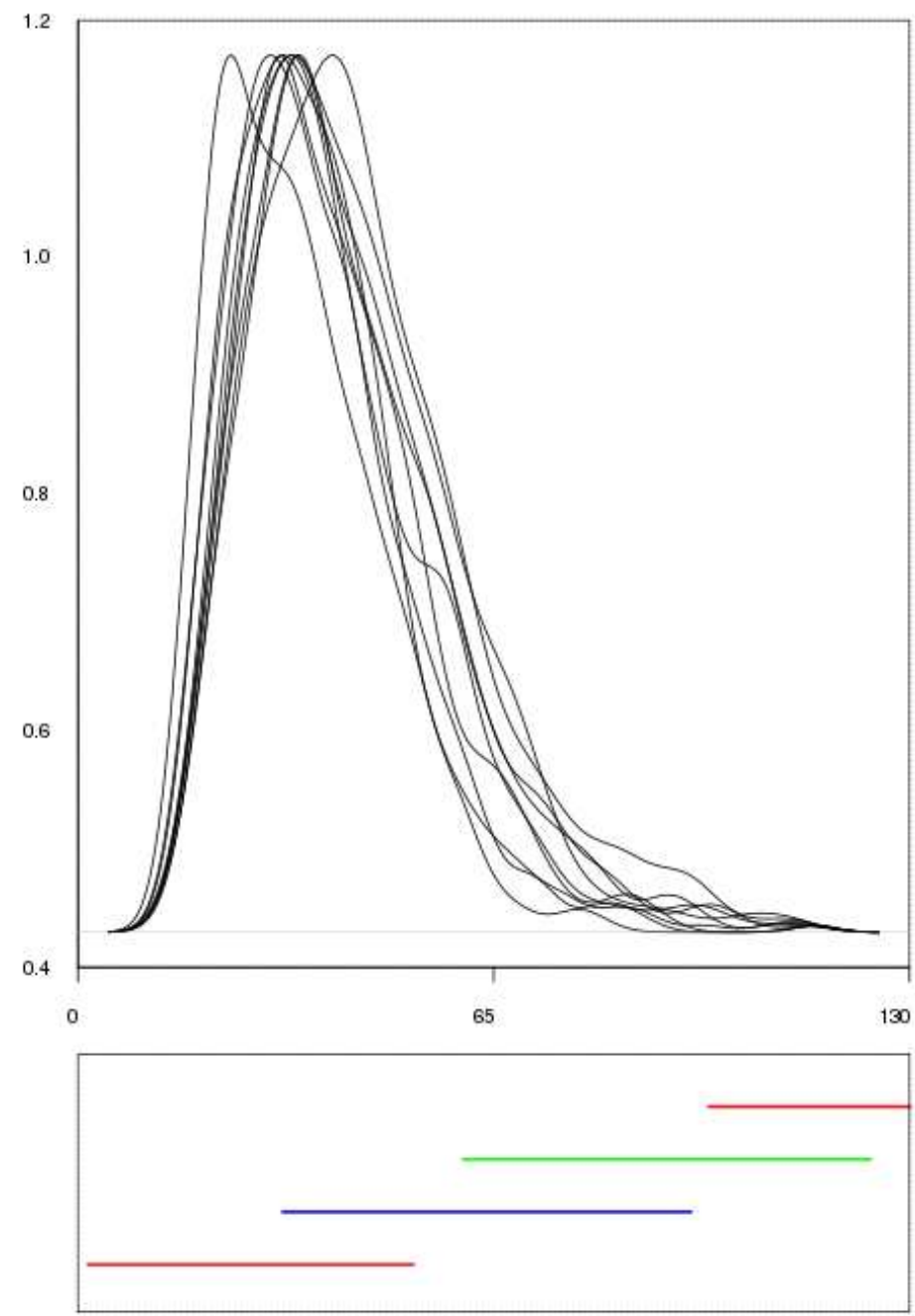

Figure 9: The ten distributions of size 500 samples (top) and the Euclidean cluster ranges (bottom) 


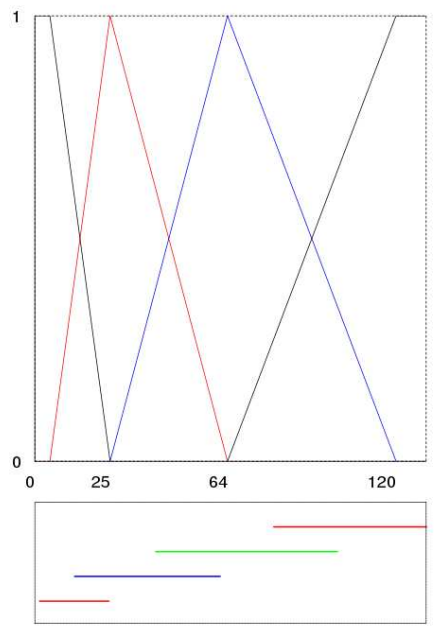

(a) TRI

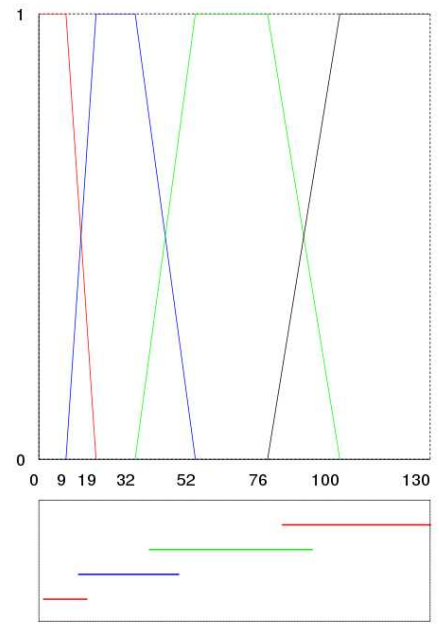

(c) K50

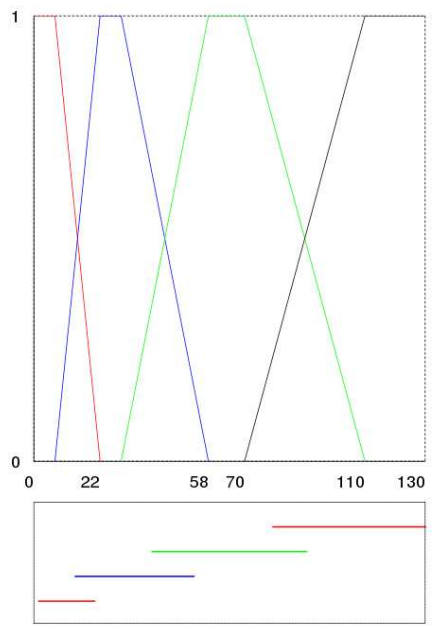

(b) K25

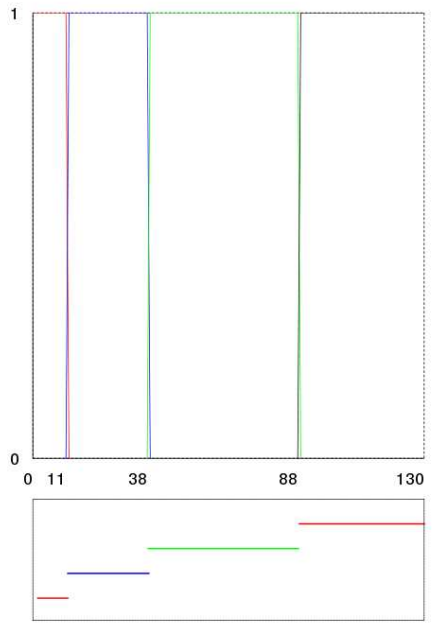

(d) K95

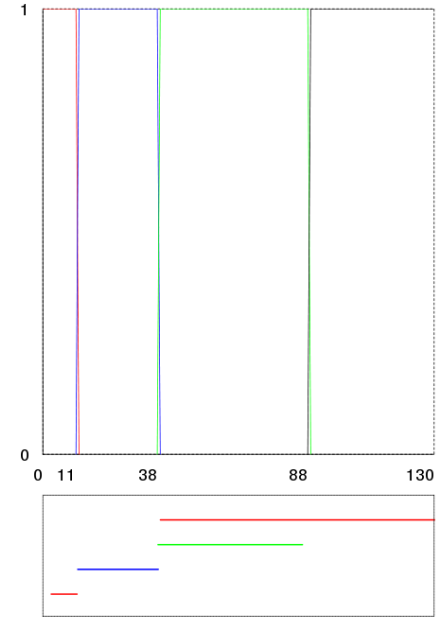

(e) K95 (size 100 sample)

Figure 10: The FPs and cluster ranges for sample size 500: (a), (b), (c), (d), (e) cluster ranges for sample size 100 and the K95 FP 
The ten sample distributions are plotted at the top of Figure 9, and the extrema of the cluster ranges, calculated over the ten runs, are plotted at the bottom. The five pseudo-metrics include the Euclidean distance and four FP-based pseudo-metrics corresponding to the FP shown in Figure 10. The cluster ranges are also plotted in Figure 10 below each corresponding FP.

We discuss these plots as follows. When the Euclidean distance is used in the hierarchical clustering, the process is completely unsupervised. The cluster bounds depend only on the number of groups and the data distribution for each run, and it is difficult to interpret them. With the FP-based pseudo-metrics, the results are different. The cluster composition reflects the knowledge represented by the FP, almost strictly for the $K 95$ partition, and with more flexibility for the $K 25, K 50$ or $T R I$ partitions. For all FPs, the extrema of the cluster bounds are located within the corresponding MF support range, reflecting the constraint that individuals belonging to the same concept are closer than those belonging to different MFs.

Table 2 summarizes the variances of the lower and upper bounds of the four clusters for each of the five pseudo-metrics.

Table 2: Variances of min and max cluster bounds, sample size: 500

\begin{tabular}{|c|c|c|c|c|c|c|c|c|c|c|}
\hline \multirow{3}{*}{ Cluster } & \multirow{2}{*}{\multicolumn{2}{|c|}{ Euclidean }} & \multicolumn{8}{|c|}{ Partition } \\
\hline & & & \multicolumn{2}{|c|}{ Tri } & \multicolumn{2}{|c|}{ K25 } & \multicolumn{2}{|c|}{ K50 } & \multicolumn{2}{|c|}{ K95 } \\
\hline & \multicolumn{2}{|c|}{$\min -\max$} & \multicolumn{2}{|c|}{$\min -\max$} & \multicolumn{2}{|c|}{$\min -\max$} & \multicolumn{2}{|c|}{$\min -\max$} & \multicolumn{2}{|c|}{$\min -\max$} \\
\hline 1 & 1 & 45 & 1 & 14 & 1 & 7 & 1 & 1 & 1 & 0 \\
\hline 2 & 47 & 129 & 14 & 48 & 6 & 22 & 1 & 15 & 0 & 0 \\
\hline 3 & 193 & 110 & 52 & 55 & 22 & 26 & 16 & 20 & 0 & 0 \\
\hline 4 & 558 & 219 & 81 & 219 & 54 & 219 & 34 & 219 & 3 & 219 \\
\hline
\end{tabular}

Table 2 exhibits a clear trend: the wider the MF kernels, the smaller is the cluster bound variance. The K95 experiment is an extreme case. This configuration drastically reduces the degree of freedom of the clustering algorithm, but it also highlights the importance of the distance parameter.

The means of the cluster counts for sample size 500 are given in Table 3 . The counts for the K95 FP may be used as a reference because this partition is close to a crisp partition. For this partition, the groups are unbalanced; few items fall in the first and the fourth cluster. This unbalance is logical because the data distribution is skewed (as shown in Figure 9). To some 
extent, the use of an FP-based pseudo-metric introduces supervision into the clustering process.

Table 3: Means of the cluster counts for sample size 500

\begin{tabular}{r|rrrrr}
\hline G & En & Tri & K25 & K50 & K95 \\
\hline 1 & 315 & 130 & 93 & 63 & 36 \\
2 & 158 & 259 & 256 & 279 & 271 \\
3 & 22 & 102 & 137 & 147 & 183 \\
4 & 4 & 10 & 14 & 12 & 11 \\
\hline
\end{tabular}

In contrast, when using the Euclidean distance, the two first groups are more numerous and the third one includes less individuals. There is no supervising effect and the data distribution is no longer reflected in the cluster composition.

However, we must be cautious regarding this point. This supervising effect remains valid under the condition that the data distribution spans the whole range of the FP. With a small sample size, certain distributions may lack items at the edge. In this case, the fourth group range may also be included in the third fuzzy set support, as is the case for the sample size 100, which is shown at the bottom of Figure 10(e). Results for sample sizes 200 and 1000 are comparable to the results given for sample size 500 .

We could therefore consider that we have a semi-supervised behavior, with the FP serving as a reference only if data are available to enforce that reference.

The study raises significant questions about the relative importance of clustering algorithm parameters and data locations for the resulting clusters. It is well known that the distance function is a key parameter for such algorithms, including the $k$-means algorithm. For instance, input variables can be weighted within the distance function, and in that case, changing the weighting coefficient vector is likely to yield a different partition.

Furthermore, the K95 experiment illustrates the importance given to expert knowledge. In the case studies, partitions that are more realistic are used to introduce expert knowledge into the clustering procedure. Thus, expert knowledge does not entirely define the clustering result, even more so in a multidimensional clustering procedure. To summarize, the results are dependent on the data and are biased by the FPs. 


\subsection{Real-world case study}

To demonstrate the value of the proposed univariate pseudo-metric, this section presents a real-world application involving geographical data and expert knowledge for decision support in wine growing.

The georeferenced data are yield data [25] from an embedded sensor on a grape-harvesting machine. The 1.4-ha field was planted with the Bourboulenc variety and was harvested in 2001 in Provence (France). The average sampling rate is approximately 2400 measurements per ha. However, because of a data acquisition problem, some records are missing. A recurrent characteristic of many agro-environmental data is their lack of regularity because of missing data and manual measurement.

The objective of the study is to identify suitable management zones based on the information found in the yield data and the domain knowledge. Several operations could then be adapted, including fertilization, winter pruning and grassing.

An algorithm that is able to process data that are not necessarily on a regular grid is used. This algorithm is derived from a region-merging algorithm, and details about the algorithm can be found in [21]. A fundamental point is the way the spatial coordinates are used here. They are not directly involved in any dissimilarity calculation but are only used to define point and zone neighborhoods. The algorithm works on two spaces simultaneously (attribute space and geographic space). The proximity criterion used for zone merging is based on dissimilarity in the attribute space and is calculated only within a given neighborhood. Spatial interpolation of data is not necessary for the algorithm to run, which is an asset because synthetic data are made by interpolation, and the artificial nature of these data is often not considered in the interpretation of the results.

The study compares the results obtained by running the algorithm with a Euclidean distance-based merging criterion and with an FP-based distance criterion. The FP is determined from local policy norms, and the fuzzy set breakpoints are 7,9,11. A FisPro [12] screenshot displaying the FP together with the data distribution is shown in Figure 11.

The algorithm yields a series of maps with a decreasing number of zones. The six zone maps are shown in Figure 12-a and 12-b.

The main difference between the two maps is the presence of a north-south structure in the FP-based map, which does not appear in the other map. Two main management zones are highlighted in 12-b, one corresponding to the 


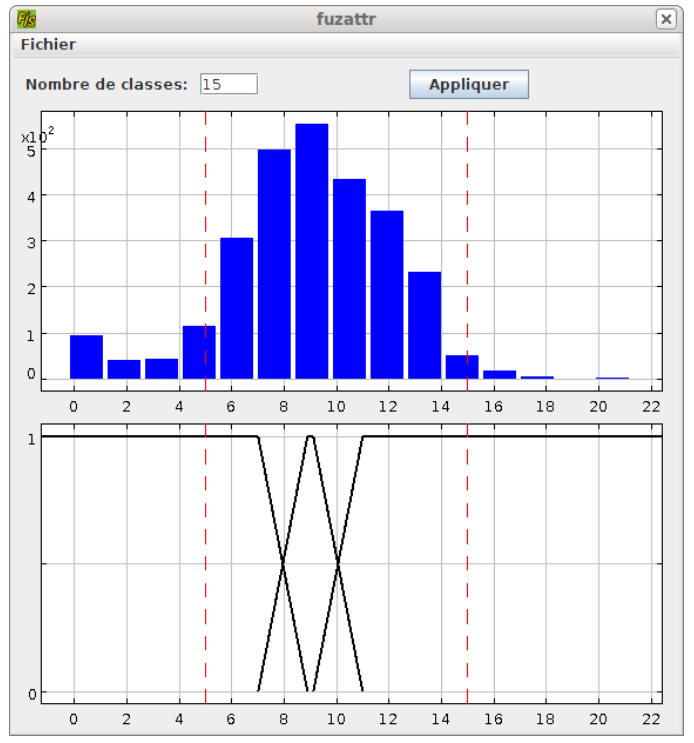

Figure 11: Histogram and FP for the yield data

northern low yield and one corresponding to the southern high yield. From an expert point of view, this makes sense. Inversely, the absence of structure in Figure 12-a is derived from the fact that, with the Euclidean distance, medium-high yield data in the south-east have been associated with lower yield data to form zone \#6 (in light gray).

A few specific zones of small size appear equally in both maps. These zones correspond to i) a zone of high yield in the center of the plot (zone \#1, in black) and ii) a few low-yield zones located along the southern edge of the field, which correspond to border effects (beginning of the rows). Depending on the goal and the machinery of the grower, these small zones may not be considered for site-specific management.

The introduction of the FP-based pseudo-metric in the zoning method provides a map that simplifies the representation of the field, according to expert knowledge, while preserving the main trends found in the available data.

In many real-world applications, multidimensional data are to be considered. We now propose a method for using FP-based pseudo-metrics in a multivariate framework. 
Author-produced version of the article published in Information Sciences, 2013, 245, 76-95.

The original publication is available at http://www.sciencedirect.com

DOI : 10.1016/j.ins.2012.07.045

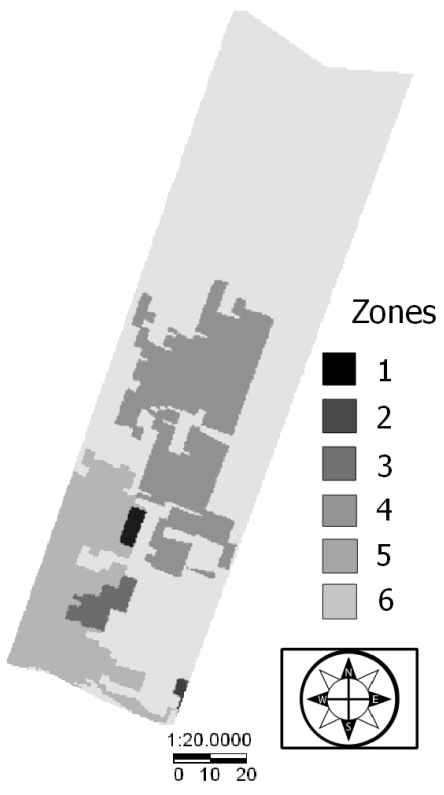

a) Euclidean distance

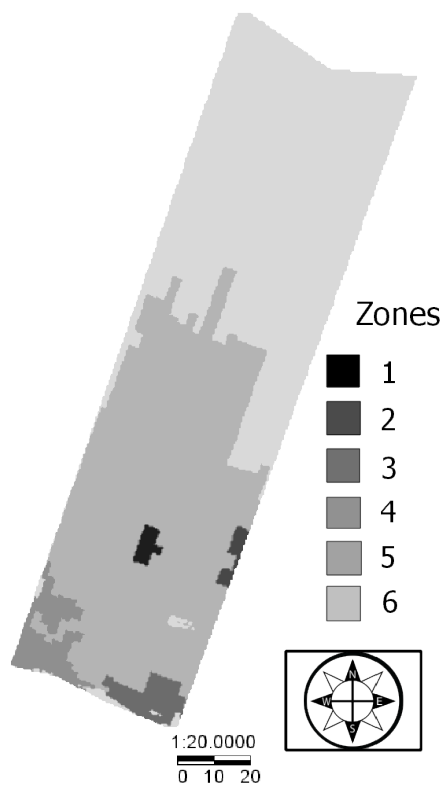

b) FP-based pseudo-metric

Figure 12: Zoning yield data with two types of distance 


\section{The multivariate case: a pseudo-metric and a case study}

The easiest way to obtain a multidimensional pseudo-metric is to perform a Minkowski-like combination of the univariate pseudo-metrics. Let two multidimensional points $x=\left(x_{1}, \ldots, x_{M}\right)$ and $y=\left(y_{1}, \ldots, y_{M}\right)$ with $x_{i}, y_{i} \in[0,1], \forall i \in 1, \ldots, M$.

We have the following definition for the pseudo-metric:

$$
\forall x, y \quad d(x, y)=\left[\sum_{j=1}^{M}\left(d_{j}\left(x_{j}, y_{j}\right)\right)^{k}\right]^{\frac{1}{k}}
$$

In Equation (13), each $d_{j}$ is a univariate distance in the $j$ th dimension, either $d_{P}$ as defined in Equation (5), which is based on a FP $P$, or a different univariate distance, for instance, a univariate Euclidean distance.

Because $d(x, y)$ is a sum of semi-distance functions, it automatically inherits the properties of a semi-distance.

For $k=2$ and regular SFPs in all dimensions, Equation (13) yields the same result as the multidimensional Euclidean distance.

Case study

Data chosen to demonstrate the value of the approach are taken from [15] and have been used previously to illustrate clustering procedures [7]. The data set describes the percentages of water, protein, fat, lactose and ash in the milk of 22 mammals. Data are given in Table 4.

The aim of our case study is to cluster the mammals, according to these five milk components, by introducing FP-based pseudo-metrics into a classical clustering procedure. First, a dissimilarity matrix between all pairs of items is computed. Then, the dissimilarity matrix is used as an input to the clustering algorithm. Because the standard $k$-means does not give stable results with the Euclidean distance, a robust version, called pam (partitioning around medoids)[17], is used. The main difference between pam and $k$-means is the definition of the cluster centers: in the robust version, the cluster centers are not computed as the mean but are necessarily data items, in a formation called a medoid. The $\mathrm{R}$ implementation[23] of pam is used in the experiments. 
Table 4: Ingredients of mammals milk

\begin{tabular}{rrrrrr}
\hline & Water & Protein & Fat & Lactose & Ash \\
\hline Bison & 86.90 & 4.80 & 1.70 & 5.70 & 0.90 \\
Buffalo & 82.10 & 5.90 & 7.90 & 4.70 & 0.78 \\
Camel & 87.70 & 3.50 & 3.40 & 4.80 & 0.71 \\
Cat & 81.60 & 10.10 & 6.30 & 4.40 & 0.75 \\
Deer & 65.90 & 10.40 & 19.70 & 2.60 & 1.40 \\
Dog & 76.30 & 9.30 & 9.50 & 3.00 & 1.20 \\
Dolphin & 44.90 & 10.60 & 34.90 & 0.90 & 0.53 \\
Donkey & 90.30 & 1.70 & 1.40 & 6.20 & 0.40 \\
Elephant & 70.70 & 3.60 & 17.60 & 5.60 & 0.63 \\
Fox & 81.60 & 6.60 & 5.90 & 4.90 & 0.93 \\
Guinea Pig & 81.90 & 7.40 & 7.20 & 2.70 & 0.85 \\
Hippo & 90.40 & 0.60 & 4.50 & 4.40 & 0.10 \\
Horse & 90.10 & 2.60 & 1.00 & 6.90 & 0.35 \\
Llama & 86.50 & 3.90 & 3.20 & 5.60 & 0.80 \\
Monkey & 88.40 & 2.20 & 2.70 & 6.40 & 0.18 \\
Mule & 90.00 & 2.00 & 1.80 & 5.50 & 0.47 \\
Orangutan & 88.50 & 1.40 & 3.50 & 6.00 & 0.24 \\
Pig & 82.80 & 7.10 & 5.10 & 3.70 & 1.10 \\
Rabbit & 71.30 & 12.30 & 13.10 & 1.90 & 2.30 \\
Rat & 72.50 & 9.20 & 12.60 & 3.30 & 1.40 \\
Reindeer & 64.80 & 10.70 & 20.30 & 2.50 & 1.40 \\
Seal & 46.40 & 9.70 & 42.00 & 0.00 & 0.85 \\
Sheep & 82.00 & 5.60 & 6.40 & 4.70 & 0.91 \\
Whale & 64.80 & 11.10 & 21.20 & 1.60 & 0.85 \\
Zebra & 86.20 & 3.00 & 4.80 & 5.30 & 0.70 \\
\hline & & & & &
\end{tabular}




\section{Using the Euclidean distance}

The results of the pam partitioning run on the multidimensional data are shown in Figure 13, which is a two-dimensional plot. The three clusters are labeled $E_{1}, E_{2}, E_{3}$.

All observations are represented by points in the plot, and principal components analysis (PCA) is used to reduce the dimensions to the two first axes. An ellipse is drawn around each cluster. The first two components of the PCA explain $94.91 \%$ of the variability, and we will study the cluster composition on the first plane, also called the principal plane. Of course, some individuals may be closer or farther on the other factorial planes.

The cluster composition is somewhat unexpected. Dog is included in the cluster of sea mammals, whereas cat and pig are assigned to a different cluster.

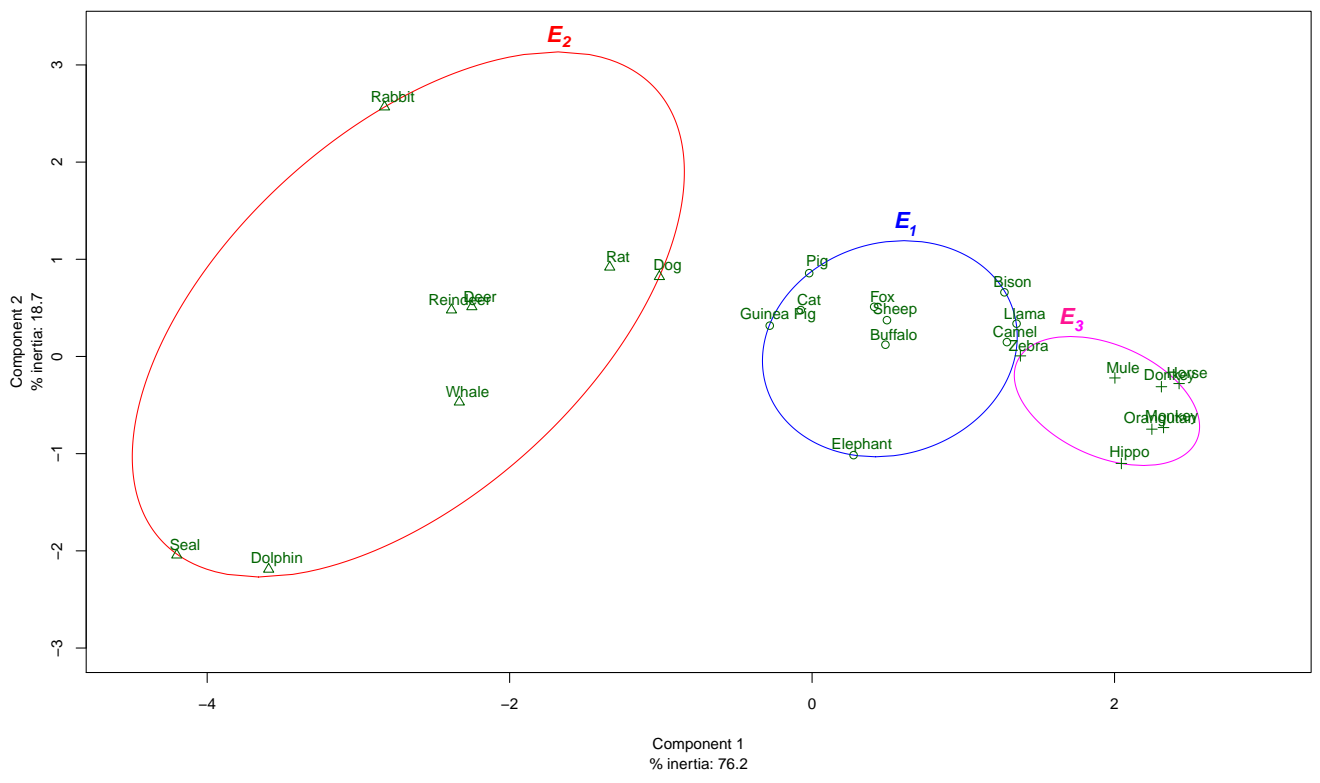

Figure 13: Clusters obtained with the Euclidean distance

A powerful indicator can be computed using the silhouette index [24]. To construct the silhouettes $S(i)$ for each item $i$, the following formula is used:

$$
S(i)=\frac{b(i)-a(i)}{\max (a(i), b(i))}
$$


where $a(i)$ is the average dissimilarity of item $i$ to all other items in the same cluster and $b(i)$ is the minimum of average dissimilarity of item $i$ to all items in other clusters. $b(i)$ can be seen as the dissimilarity between item $i$ and its neighbor cluster, i.e., the nearest cluster to which it does not belong. The average silhouette $S_{c}$ for each cluster is simply the average of the $S(i)$ for all items in the $c t h$ cluster. Similarly, the overall average silhouette $\bar{S}$ is the average of the $S(i)$ for all items in the whole data set.

Figure 14 shows the silhouette values corresponding to the clustering using the Euclidean distance. Three of the silhouette values are negative: llama, camel and dog. Others, like the rat-related silhouette value, are close to zero.

The silhouette index is based on cluster tightness and separation and is derived from the formula $-1 \leq S(i) \leq 1$. A value close to one indicates that the observation is correctly assigned to a group; a small value, even more so a negative one, results in a wrong assignment. The largest overall average silhouette indicates the best clustering.

Silhouette index values resulting from the Euclidean-based clustering are given in the second row of Table $5, S_{c}$ for each cluster and $\bar{S}$ for the overall averaged value. The same calculations will be performed for the SFP-based pseudo-metric.

Table 5: Averaged silhouettes for each cluster and averaged overall value, Euclidean and FP-based pseudo-metrics

\begin{tabular}{c|c|c|c|c}
\hline \multirow{2}{*}{ Euclidean } & $E_{1}$ & $E_{2}$ & $E_{3}$ & Overall \\
\cline { 2 - 5 } & 0.27 & 0.28 & 0.55 & 0.35 \\
\hline \multirow{2}{*}{ FP-based } & $P_{1}$ & $P_{2}$ & $P_{3}$ & Overall \\
\cline { 2 - 5 } & 0.62 & 0.30 & 0.57 & 0.48 \\
\hline
\end{tabular}

\section{Introducing expertise by fuzzy partitions}

The proposed pseudo-metric allows for the introduction of expert knowledge by FPs. Two variables are considered, Water and Fat. The other three variables are addressed using the Euclidean distance.

Figure 15(b) displays the FP corresponding to low and high Water content. 


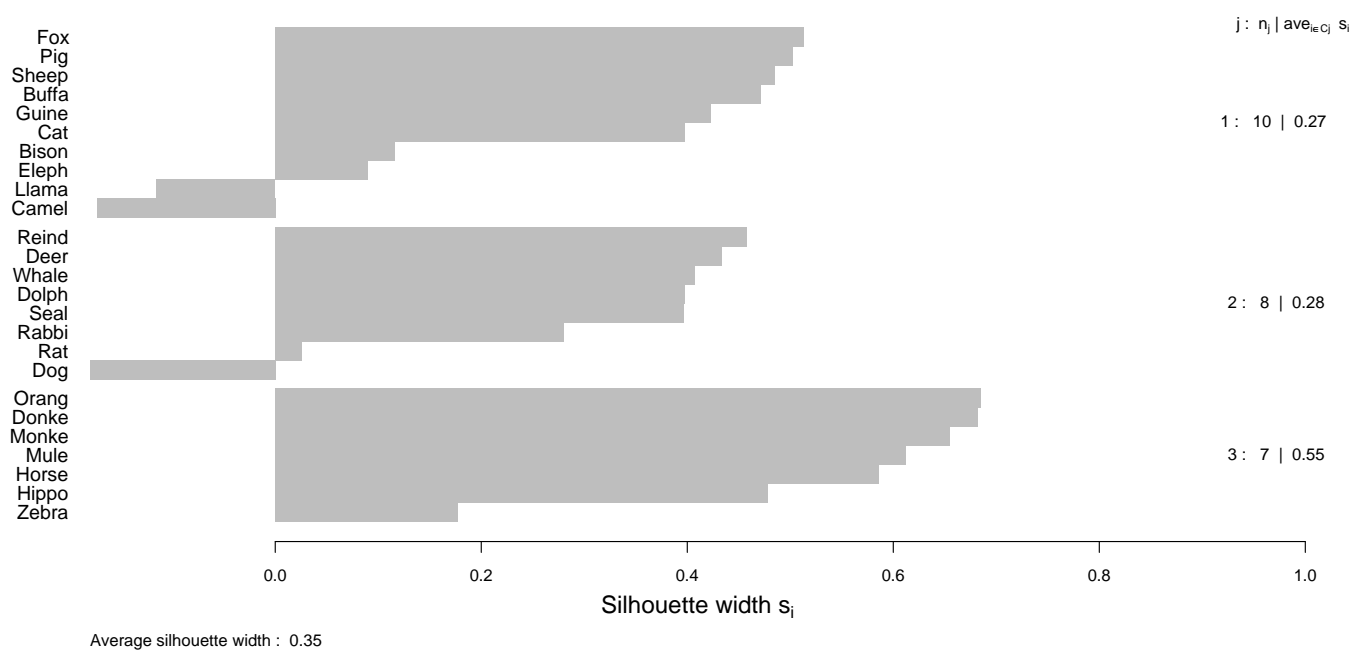

Figure 14: Silhouettes with Euclidean distance

The Fat content variable has been partitioned into four groups as shown in Figure 15(a). This number of groups is motivated by the dispersion of the data distribution for the variables. The ratio of the standard deviation to the mean is approximately 0.16 for Water content, whereas it is higher than 1 for this variable.

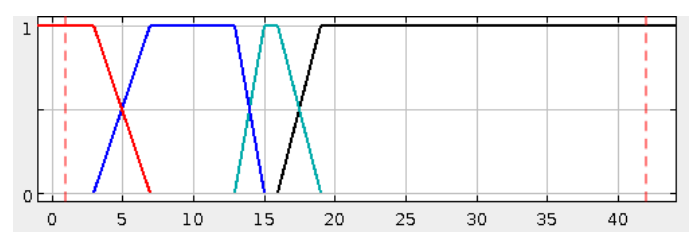

(a) Fat

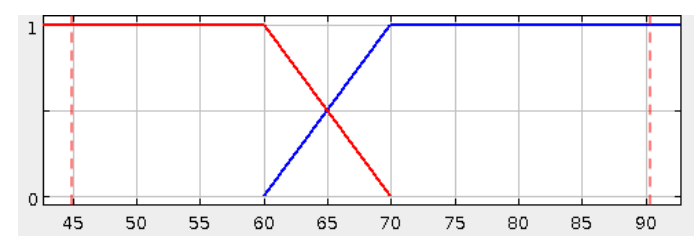

(b) Water

Figure 15: Expert FPs

Expertise in MF design consists, for instance, of considering that above 20\%, the Fat content is definitely high. It is well known that marine mammals milk has a much higher Fat content $(\geq 20 \%)$ than terrestrial mammals milk and that variations between $20 \%$ and $42 \%$ do not have much significance. 


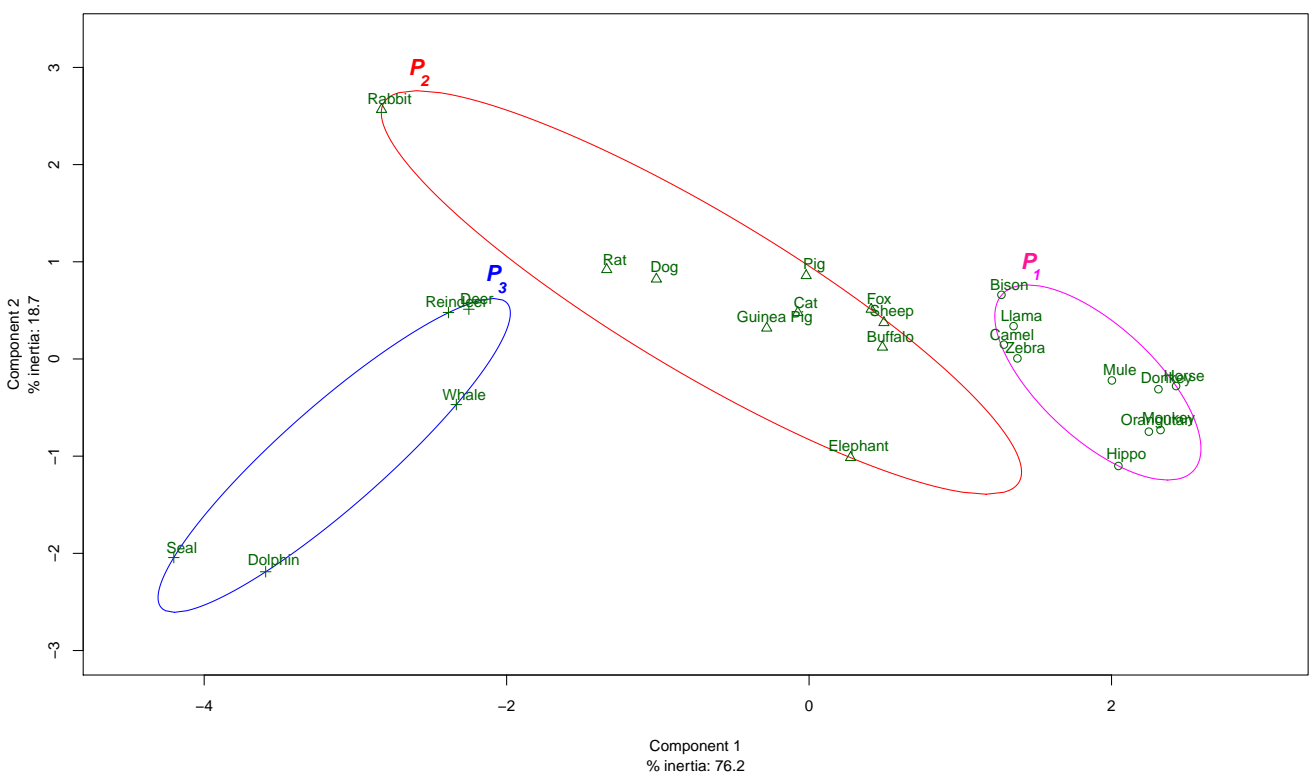

Figure 16: Clusters obtained with the FP-based pseudo-metric

The new silhouette indices are given in the fourth row of Table 5. These indices also support the SFP-based partitions: all $S_{c}$ values are higher, and the overall average $\bar{S}$ has been improved to 0.48 from 0.35 .

The three new clusters are shown in Figure 16 and are labeled $P_{1}, P_{2}$ and $P_{3}$. As could be expected, the cluster shapes are sensitive to the metric. When comparing Figure 16 with Figure 13, changes are present for all of the clusters. Pig, dog and cat are now together in the central group. The boundary between the central and right clusters has also been modified. These two clusters are more neatly separated according to the plot. Bison, camel and llama are now in the group on the right, together with zebra. The new silhouettes are plotted in Figure 17 and show an improvement compared to the silhouettes plotted in Figure 14.

\section{Conclusion}

In this paper, a univariate semi-distance function was introduced to reflect the semantics of FPs defined on numerical domains to incorporate domain 


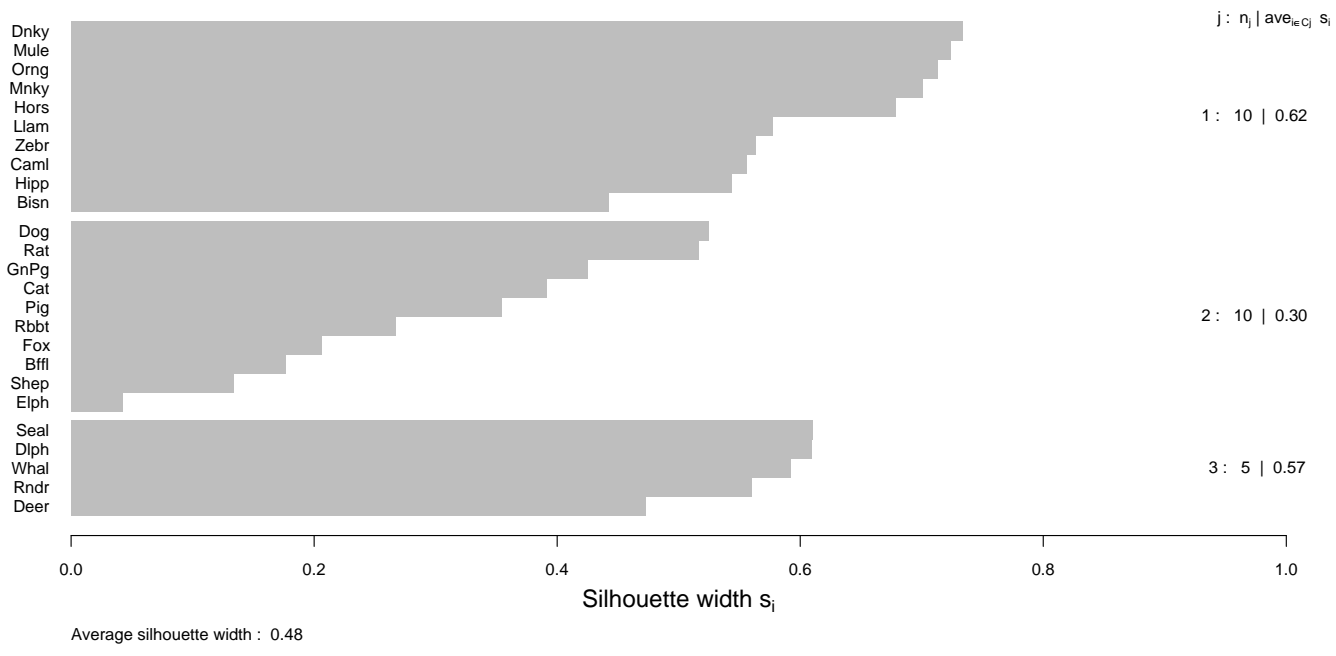

Figure 17: Silhouettes with the FP-based pseudo-metric

knowledge into the calculations. This function is called an FP-based pseudometric.

To define such a pseudo-metric for a given dimension, a FP must be specified for the corresponding linguistic variable. The pseudo-metric then involves a combination of symbolic and numerical terms, which allows for the pseudo-metric to distort the Euclidean distance according to the FP structure.

The numerical term considers multiple membership in transition zones between concepts. The symbolic term factors in the distance between concepts. Each concept is associated with an MF within the partition. All concepts are considered as equidistant, independent of the Euclidean distance between kernel points. All points within a given kernel are assumed to have a null distance because they are prototypes of the same concept.

The proposed FP-based univariate pseudo-metric behavior has been studied with synthetic data sets to investigate its behavior and the ways it differs from the Euclidean distance. The practical use of the proposed pseudo-metric has been shown by introducing it into a segmentation algorithm to design management zones in cultivated fields.

A multivariate semi-distance was proposed as a Minkowski-like combina- 
tion of univariate semi-distances that can freely use different types of univariate distances or semi-distances. For instance, the multivariate semi-distance can associate a Euclidean distance in one dimension with an FP-based semidistance in another dimension.

The multivariate FP-based pseudo-metric was applied to a real-world clustering case in the field of Biology: mammals milk. The results show the effect of the distortion of the Euclidean space on two of the five available variables. They highlight the clusters sensitivity to the distance choice. The new clusters are better separated and are likely to be more interpretable.

Let us point out that independent of their imprecision, real-world numerical data may have some degree of imperfection because of their incomplete representativeness for the problem to be modeled. This is often the case in clustering and especially so in Biology because of the high variability of living organisms. Integration of expert knowledge can overcome this difficulty, and this is the aim of the present approach.

Targeted real-world applications include merging processes in image analysis (region growing algorithms) and statistical procedures (hierarchical clustering) in any application area in which knowledge integration is important. Using the new semi-distance in various application domains should draw attention to its potential for incorporating expert knowledge into learning methods. Further work could also be conducted to define pseudo-metrics on multidimensional FPs.

\section{References}

[1] J. C. Bezdek, Pattern Recognition with Fuzzy Objective Functions Algorithms, Plenum Press, New York, 1981.

[2] I. Bloch, On fuzzy distances and their use in image processing under imprecision, Pattern Recognition 32 (1999) 1873-1895.

[3] S. Boriah, V. Chandola, V. Kumar, Similarity measures for categorical data: A comparative evaluation, in: SIAM Data Mining Conference, Atlanta, GA, 2008, pp. 243-254.

[4] B. B. Chaudhuri, A. Rosenfeld, On a metric distance between fuzzy sets, Pattern Recognition Letters 17 (1996) 1157-1160.

[5] C. Coppola, T. Pacelli, Approximate distances, pointless geometry and incomplete information, Fuzzy Sets and Systems 157 (2006) 2371-2383. 
[6] P. Diamond, P. Kloeden, Metric spaces of fuzzy sets, Fuzzy Sets and Systems 35 (1990) 241-249.

[7] W. J. Dixon, BMDP statistical software manual: to accompany the 1990 software release, BDMP (1990).

[8] D. Dubois, H. Prade, Fuzzy Sets and Systems: Theory and Applications, Academic Press, New York, 1980.

[9] L. Egghe, R. Rousseau, Classical retrieval and overlap measures satisfy the requirements for rankings based on a lorenz curve, Information Processing and Management 42 (2006) 106-120.

[10] J. Fan, W. Xie, Distance measure and induced fuzzy entropy, Fuzzy Sets and Systems 104 (1999) 305-314.

[11] S. Guillaume, B. Charnomordic, Generating an interpretable family of fuzzy partitions, IEEE Transactions on Fuzzy Systems 12 (3) (2004) $324-335$.

[12] S. Guillaume, B. Charnomordic, Learning interpretable fuzzy inference systems with fispro, Information Sciences 181 (2011) 4409-4427.

[13] S. Guillaume, B. Charnomordic, P. Loisel, A numerical distance based on fuzzy partitions, in: S. Galichet, J. Montero, G. Mauris (eds.), Proceedings of the 7th conference of the European Society for Fuzzy Logic and Technology (EUSFLAT-2011) and LFA-2011, Annecy, France, 2011, pp. 1000-1006.

[14] R. E. Hammah, J. H. Curran, On distance measures for the fuzzy kmeans algorithm for joint data, Rock Mechanics and Rock Engineering 32 (1) (1999) 1-27.

[15] J. A. Hartigan, Clustering Algorithms, Wiley, 1975.

[16] A-L. Jousselme, P. Maupin. Distances in evidence theory: Comprehensive survey and generalizations. International Journal of Approximate Reasoning, 53(2):118 - 145, 2012.

[17] L. Kaufman, P. Rousseeuw, Finding Groups in Data: An Introduction to Cluster Analysis, Wiley Interscience, New York, 1990. 
[18] J. Liang, R. Li, Y. Qian. Distance: A more comprehensible perspective for measures in rough set theory. Knowledge-Based Systems, 27(0):126 $-136,2012$.

[19] R. Lowen, W. Peeters, Distance between fuzzy sets representing grey level images, Fuzzy Sets and Systems 99 (1998) 135-149.

[20] G. Mansingh, K-M. Osei-Bryson, H. Reichgelt. Using ontologies to facilitate post-processing of association rules by domain experts. Information Sciences, 181(3):419 - 434, 2011.

[21] M. Pedroso, J. Taylor, B. Tisseyre, B. Charnomordic, S. Guillaume, A segmentation algorithm for the delineation of management zones, Computer and Electronics in Agriculture 70 (2010) 199-208.

[22] W. Pedrycz, Why triangular membership functions?, Fuzzy sets and Systems 64 (1) (1994) 21-30.

[23] R Development Core Team, R: A Language and Environment for Statistical Computing, R Foundation for Statistical Computing, Vienna, Austria, ISBN 3-900051-07-0 (2008).

URL http://www.R-project.org

[24] P. J. Rousseeuw, Silhouettes: a graphical aid to the interpretation and validation of cluster analysis, Journal of Computational and Applied Mathematics 20 (1987) 53-65.

[25] B. Tisseyre, A. B. McBratney, A technical opportunity index based on mathematical morphology for site-specific management: an application to viticulture, Precision Agriculture 9 (2008) 101-113.

[26] V. Torra, Y. Narukawa. On a comparison between Mahalanobis distance and Choquet integral: The Choquet Mahalanobis operator. Information Sciences, 190(0):56 - 63, 2012.

[27] W. Trutschnig, G. González-Rodríguez, A. Colubi, M. A. Gil, A new family of metrics for compact, convex (fuzzy) sets based on a generalized concept of mid and spread, Information Sciences 179 (23) (2009) 3964 3972 . 
[28] Z. Xu, J. Chen, An overview of distance and similarity measures of intuitionistic fuzzy sets, International Journal of Uncertainty, Fuzziness and Knowledge-Based Systems 16 (2008) 529-555.

[29] Z. Xu, M. Xia, Distance and similarity measures for hesitant fuzzy sets, Information Sciences 181 (2010) 2128-2138.

[30] L. A. Zadeh, The concept of linguistic variable and its application to approximate reasoning - parts i, ii and iii, Information Sciences 8-9 (1975) 199-249,301-357,43-80.

[31] W. Zeng, P. Guo, Normalized distance, similarity measure, inclusion measure and entropy of interval-valued fuzzy sets and their relationship, Information Sciences 178 (2008) 1334-1342. 